\title{
A Critical Neurodevelopmental Role for L-Type Voltage-Gated Calcium Channels in Neurite Extension and Radial Migration
}

\author{
Satoshi Kamijo, ${ }^{1}$ Yuichiro Ishii, ${ }^{1}$ Shin-ichiro Horigane, ${ }^{1,2}$ Kanzo Suzuki, ${ }^{1}$ Masamichi Ohkura, ${ }^{3}$ Junichi Nakai, ${ }^{3}$ \\ Hajime Fujii, ${ }^{1}$ Sayaka Takemoto-Kimura, ${ }^{2,4}$ and ${ }^{-}$Haruhiko Bito ${ }^{1,5}$ \\ ${ }^{1}$ Department of Neurochemistry, Graduate School of Medicine, The University of Tokyo, Bunkyo-ku, Tokyo 113-0033, Japan, ${ }^{2}$ Department of Neuroscience \\ I, Research Institute of Environmental Medicine, Nagoya University, Chikusa-ku, Nagoya 464-8601, Japan, ${ }^{3}$ Brain and Body System Science Institute and \\ Graduate School of Science and Engineering, Saitama University, Sakura-ku, Saitama City, Saitama 338-8570, Japan, ${ }^{4}$ PRESO-JST, Chiyoda-ku, Tokyo \\ 102-0076, Japan, and 5International Research Center for Neurointelligence, The University of Tokyo, Bunkyo-ku, Tokyo 113-0033, Japan
}

Despite many association studies linking gene polymorphisms and mutations of L-type voltage-gated $\mathrm{Ca}^{2+}$ channels (VGCCs) in neurodevelopmental disorders such as autism and schizophrenia, the roles of specific L-type VGCC during brain development remain unclear. Calcium signaling has been shown to be essential for neurodevelopmental processes such as sculpting of neurites, functional wiring, and fine tuning of growing networks. To investigate this relationship, we performed submembraneous calcium imaging using a membranetethered genetically encoded calcium indicator (GECI) Lck-G-CaMP7. We successfully recorded spontaneous regenerative calcium transients (SRCaTs) in developing mouse excitatory cortical neurons prepared from both sexes before synapse formation. SRCaTs originated locally in immature neurites independently of somatic calcium rises and were significantly more elevated in the axons than in dendrites. SRCaTs were not blocked by tetrodoxin, a $\mathrm{Na}^{+}$channel blocker, but were strongly inhibited by hyperpolarization, suggesting a voltagedependent source. Pharmacological and genetic manipulations revealed the critical importance of the $\mathrm{Ca}_{\mathrm{v}} 1.2$ (CACNA1C) pore-forming subunit of L-type VGCCs, which were indeed expressed in immature mouse brains. Consistently, knocking out $\mathrm{Ca}_{\mathrm{v}} 1.2$ resulted in significant alterations of neurite outgrowth. Furthermore, expression of a gain-of-function $\mathrm{Ca}_{\mathrm{v}} 1.2$ mutant found in Timothy syndrome, an autosomal dominant multisystem disorder exhibiting syndromic autism, resulted in impaired radial migration of layer $2 / 3$ excitatory neurons, whereas postnatal abrogation of $\mathrm{Ca}_{\mathrm{v}} 1.2$ enhancement could rescue cortical malformation. Together, these lines of evidence suggest a critical role for spontaneous opening of L-type VGCCs in neural development and corticogenesis and indicate that L-type VGCCs might constitute a perinatal therapeutic target for neuropsychiatric calciochannelopathies.

Key words: calcium channel; calcium signaling; circuit development; radial migration; spontaneous activity

Significance Statement

Despite many association studies linking gene polymorphisms and mutations of L-type voltage-gated $\mathrm{Ca}^{2+}$ channels (VGCCs) in neurodevelopmental disorders such as autism and schizophrenia, the roles of specific L-type VGCCs during brain development remain unclear. We here combined the latest $\mathrm{Ca}^{2+}$ indicator technology, quantitative pharmacology, and in utero electroporation and found a hitherto unsuspected role for L-type VGCCs in determining the $\mathrm{Ca}^{2+}$ signaling landscape of mouse immature neurons. We found that malfunctional L-type VGCCs in immature neurons before birth might cause errors in neuritic growth and cortical migration. Interestingly, the retarded corticogenesis phenotype was rescued by postnatal correction of L-type VGCC signal aberration. These findings suggest that L-type VGCCs might constitute a perinatal therapeutic target for neurodevelopment-associated psychiatric disorders.

\section{Introduction}

Neurons change their location and morphology dynamically during brain development to form functional neural circuits. Although many developmental signaling pathways have been doc-

Received Aug. 16, 2017; revised Dec. 28, 2017; accepted April 21, 2018.

Author contributions: S.K., H.F., S.T.-K., and H.B. designed research; S.K., Y.I., S.H., and K.S. performed research; M.O., and J.N. contributed unpublished reagents/analytic tools; S.K., Y.I., H.F., and S.T.-K. analyzed data; S.K., H.F., and H.B. wrote the paper. umented, the signal transduction regulation by calcium remains of greatest interest because it is ubiquitous, versatile, and activitydependent (Spitzer, 2006). Previous studies showed that calcium signaling can modulate the length of neurites in various types of 
neurons (Gomez and Spitzer, 1999; Wayman et al., 2008; Takemoto-Kimura et al., 2010), control growth cone motility (Zheng, 2000; Henley and Poo, 2004), and sculpt the dendritic arborization in fruit flies (Kanamori et al., 2013). Many candidate molecules acting downstream of calcium such as calcium/ calmodulin-dependent protein kinase (CaMK), protein kinase $\mathrm{C}$, and calcineurin have been shown to play critical roles in morphological remodeling processes (Cabell and Audesirk, 1993; Lautermilch and Spitzer, 2000; Ageta-Ishihara et al., 2009). We found that different subspecies within the same CaMKI family, CaMKI $\alpha$ and CaMKI $\gamma$, regulated the length of axons and dendrites, respectively, downstream of various upstream signaling molecules such as brain-derived neurotrophic factor, excitatory GABA, or Wnt5a (Takemoto-Kimura et al., 2007; Ageta-Ishihara et al., 2009; Takemoto-Kimura et al., 2010; Horigane et al., 2016). Therefore, distinct downstream effector molecules may sense changes in intracellular calcium concentrations and act as differential decoders of the local "calcium codes" (Fujii et al., 2013).

What, then, are the critical $\mathrm{Ca}^{2+}$ signals and $\mathrm{Ca}^{2+}$ sources that are triggered to elevate $\mathrm{Ca}^{2+}$ within a population of soon-to-bewired, immature neurons? Although developing neurons have spontaneous fluctuations in intracellular calcium concentration (Tang et al., 2003), the molecular details of how these intracellular $\mathrm{Ca}^{2+}$ changes occur remain unsolved. Recent findings from several genome-wide association studies (GWAS) suggest an important role for calcium signaling molecules such as voltage-gated calcium channels (VGCCs) in the etiology and susceptibility underlying neurodevelopmental psychiatric diseases such as autism and schizophrenia (Schizophrenia Psychiatric Genome-Wide Association Study (GWAS) Consortium, 2011; Cross-Disorder Group of the Psychiatric Genomics Consortium, 2013; De Rubeis et al., 2014; Purcell et al., 2014). However, how malfunction of VGCCs could contribute to miswiring of cortical circuits, for example, is not known. Therefore, a deeper understanding of the role of VGCCs during neuronal development is required.

To address this question, here, we introduced a membranetethered genetically encoded calcium indicator (GECI) Lck-GCaMP7 combined with in utero electroporation. Lck-G-CaMP7 imaging enabled us to identify and record Spontaneous Regenerative Calcium Transients (SRCaTs) in developing excitatory cortical neurons with high signal-to-noise ratio. Axonal SRCaTs were significantly larger than dendritic SRCaTs. Pharmacological screening revealed that SRCaTs were membrane voltagedependent and their calcium influx was mediated by the L-type VGCCs $\mathrm{Ca}_{\mathrm{v}} 1.2$ (CACNA1C) and $\mathrm{Ca}_{\mathrm{v}} 1.3$ (CACNA1D). Consistently, in developing neurons, knocking out L-type VGCCs resulted in shorter neurites. We also found that, during perinatal development, introducing a gain-of-function mutation of L-type calcium channels impaired cortical radial migration, whereas reversing this manipulation postnatally was sufficient to rescue normal corticogenesis. Together, these results underscore hitherto un-

Research Program for Brain Sciences Grant to S.T.-K. and H.F.); the Japan Foundation for Applied Enzymology, the Takeda Science Foundation, the Hitachi Global Foundation, and the Nakatani Foundation (to H.B.). S.K., S.H., and K.S. were supported by JSPS predoctoral fellowships. We thank R.Y. Tsien (UCSD) for mCherry CDNA; llya B. Bezprozvanny (UTSW) for sHA-Cav1.2-T1039Y and HA-Cav1.3-T1033Y cDNAs; Feng Zhang (MIT) for hSpCas9 cDNA (through Addgene); Y. Kondo, H. Yamashige, and T. Kinbara for assistance; and all members of the Bito and Takemoto-Kimura laboratories for discussion throughout the project.

The authors declare no competing financial interests.

Correspondence should be addressed to Haruhiko Bito, Department of Neurochemistry, Graduate School of Medicine, The University of Tokyo, Igakubu-3-gokan Room S606, 7-3-1 Hongo, Bunkyo-ku, Tokyo 113-0033, Japan. E-mail: hbito@m.u-tokyo.ac.jp.

DOI:10.1523/JNEUROSCI.2357-17.2018

Copyright $\odot 2018$ the authors $\quad 0270-6474 / 18 / 385552-15 \$ 15.00 / 0$ suspected and crucial developmental impacts of perinatally expressed L-type VGCC signaling in neurodevelopment.

\section{Materials and Methods}

Animals. ICR mice and C57BL/6J mice were purchased from Japan SLC. Conditional Cacnalc $\left(\mathrm{Ca}_{\mathrm{v}} 1.2\right)$ knock-out mice Cacnalc ${ }^{\mathrm{tm} 3 \mathrm{Hfm}} / \mathrm{J}$ (Jax stock number 024714, http://www.jax.org/strain/024714, RRID:IMSR_JAX: 024714) were obtained from The Jackson Laboratory (Seisenberger et al., 2000). Conditional knock-out mice were bred with C57BL/6J (RRID: IMSR_JAX:000664) mice and genotypes were verified by genome PCR following the manufacturer's (The Jackson Laboratory) genotyping protocol. ICR mice (RRID:IMSR_TAC:icr) were used in all experiments except for that shown in Figure 6. We used embryos and pups of both sexes. All animal experiments were approved by the animal experiment committee of The University of Tokyo Graduate School of Medicine.

Plasmid construction. To obtain Lck-G-CaMP7, membrane targeting $\mathrm{N}$-terminal peptide from human Lck tyrosine kinase was attached to the $\mathrm{N}$ terminus of G-CaMP7 (Ohkura et al., 2012) using the In-Fusion HD cloning kit (TaKaRa). The fused sequence was subcloned into the downstream of CAG promoter. Other Lck-GCaMPs were made in the same manner. GCaMP6m and GCaMP6s were purchased from Addgene (40753, 40754). All plasmids used in this study were CAG-promoter driven to evade developmental gene suppression by methylation unless otherwise stated (Tabata and Nakajima, 2001). mCherry-KRasCT was made by attaching the CAAX motif of K-Ras to the C terminus of mCherry. WT mouse inward rectifier potassium channel $\mathrm{K}_{\mathrm{ir}} 2.1$ was subcloned and hyperpolarization-deficient V302M mutation was introduced by sitedirected mutagenesis (Bendahhou et al., 2003, Ma et al., 2007). By fusing WT $\mathrm{K}_{\mathrm{ir}} 2.1$ or V302M mutant to mCherry-KRasCT and Lck-G-CaMP7 flanked by T2A and P2A self-cleaving peptides, we obtained Lck-GCaMP7-T2A-K $\mathrm{K}_{\mathrm{ir}}$ 2.1-P2A-mCherry-KRasCT.

Rat sHA-Ca $1.2-\mathrm{T} 1039 \mathrm{Y}$ and HA-Ca $1.3-\mathrm{T} 1033 \mathrm{Y}$ channels were kind gifts from Dr. Bezprozvanny (Zhang et al., 2006). They contain a hemagglutinin (HA) sequence in the extracellular loop for $\mathrm{Ca}_{\mathrm{v}} 1.2$ and the $\mathrm{N}$ terminus for $\mathrm{Ca}_{\mathrm{v}} 1.3$. The $\mathrm{N}$ terminus of $\mathrm{Ca}_{\mathrm{v}} 1.3$ was replaced with that of $\mathrm{Ca}_{\mathrm{v}} 2.2$ and both $\mathrm{Ca}_{\mathrm{v}} 1.2$ and $\mathrm{Ca}_{\mathrm{v}} 1.3$ harbor dihydropyridine (DHP)insensitive point mutation T1039Y for $\mathrm{Ca}_{\mathrm{v}} 1.2$ and T1033Y for $\mathrm{Ca}_{\mathrm{v}} 1.3(\mathrm{He}$ et al., 1997). Timothy syndrome G406R gain-of-function mutation (Barrett and Tsien, 2008) and 4EQ $\mathrm{Ca}^{2+}$-permeability-reducing mutations (Parent and Gopalakrishnan, 1995) were introduced into the partial sequence of $\mathrm{Ca}_{\mathrm{v}} 1.2$ by site-directed mutagenesis. For pharmacological knock-out experiments, a DHP-insensitive mutation was corrected to WT residue and used as a DHP-sensitive control. After the mutations were verified by sequencing, the mutated fragments were put back into the original pCAG-sHA-Ca $1.2-\mathrm{T} 1039 \mathrm{Y}$ plasmid. To enhance surface expression of exogenous calcium channels, we coexpressed rat $\mathrm{Ca}_{\mathrm{v}} \alpha 2 \delta 1$ and $\mathrm{Ca}_{\mathrm{v}} \beta 3$ auxiliary subunits (Addgene, 26575, 26574). These two subunits were fused to self-cleaving sequences and transfection marker mCherry-KRasCT to obtain pCAG-Ca $\alpha 2 \delta 1-\mathrm{T} 2 \mathrm{~A}-\mathrm{Ca}_{\mathrm{v}} \beta 3-\mathrm{P} 2 \mathrm{~A}-$ mCherry-KRasCT. This plasmid is simply referred to auxiliary subunits herein. The obtained plasmid contained a conservative mutation (Gto-A rendering an $\mathrm{R}$ into $\mathrm{K}$ ) at position 1087 of $\mathrm{Ca}_{\mathrm{v}} \alpha 2 \delta 1,5$ aa away from the C-terminal end and remote from structural determinants of $\alpha 2 \delta$ subunit which may contribute to surface calcium channel expression (Simms and Zamponi, 2014), such as the von Willebrand factor domain or a hydrophobic stretch near a putative palmitoylation site. Consistently, whereas $\mathrm{R}$ is a common residue at this position across many species, we note that $\mathrm{R}$ may be substituted by $\mathrm{K}$ in some mammalian species [Microcebus murinus (XP_020145390.1), Cricetulus griseus (XP_016832660.1), Galeopterus variegatus (XP_008566360.1), Erinaceus europaeus (XP_016045794.1)] or amphibian species [Rana catesbeiana (BAB18554.1), Nanorana parkeri (XP_018419067.1)], whereas R is also substituted by $\mathrm{T}$ in primates [Homo sapiens (XP_006716181.1), Pan troglodytes (XP_009451727.1), Pan paniscus (XP_008974571.1), Pongo abelii (NP_001124862.1)].

For doxycycline experiments, we made pCAG-tTA3G or pCAG-rtTA3G (Tet-off and Tet-on, Clontech, with consent from TET Systems). Calcium channels were subcloned into downstream of TRE promoter to obtain pTRE-Tight-sHA-Ca $\mathrm{v}_{\mathrm{v}} 1.2-\mathrm{T} 1039 \mathrm{Y}$ and its mutants. 
To excise floxed exons in conditionally floxed $\mathrm{Ca}_{\mathrm{v}} 1.2$ mice (Jax stock number 024714), we transfected pCAG-Cre-P2A-mCherry-KRasCT, which was constructed using In-Fusion HD cloning method. The recombination activity was confirmed by coexpression with floxedGFP reporter plasmid in HeLa cells. For SLENDR experiments, we used pX330-U6-Chimeric_BB-CBh-hSpCas9 (Addgene, 42230) and a gRNA sequence against $\mathrm{Ca}_{\mathrm{v}} 1.2$ as described previously (Mikuni et al., 2016). A 200 bp single-stranded donor DNA for $\mathrm{Ca}_{\mathrm{v}} 1.2$ editing and $\mathrm{HA}$ insertion was electroporated into the embryos of pregnant C57BL/6J mice with Cas9 plasmid at E12.5.

In utero electroporation. Basic experimental procedures were the same as those described previously (Ageta-Ishihara et al., 2009; Horigane et al., 2016). Pregnant embryonic day 12.5 (E12.5) or E15.5 ICR mice (Japan SLC) were anesthetized with $90 \mathrm{mg} / \mathrm{kg}$ pentobarbital (Kyoritsu Seiyaku). Then, a 2-4 $\mu \mathrm{g} / \mu \mathrm{l}$ DNA solution was injected into the lateral ventricle with a custom-made glass pipette $(1.5 \mathrm{~mm}$ outer diameter, $1.17 \mathrm{~mm}$ inner diameter; Harvard Apparatus). The injected embryo was pinched with 5-mm-diameter custom-made electrodes (BEX) and electrical pulses were applied in the following conditions: for E15.5 embryos: pulse number $5 \times 2$ trains, amplitude $45 \mathrm{~V}$, duration $50 \mathrm{~ms}$; for E12.5 embryos: pulse number $7 \times 1$ train, amplitude $40 \mathrm{~V}$, duration $50 \mathrm{~ms}$. Electrical pulses were generated by ECM 830 electroporator (BTX; Harvard Apparatus). In utero electroporation into $\mathrm{C} 57 \mathrm{BL} / 6 \mathrm{~J}$ or conditional knock-out mice was performed in the same manner.

Cortical cell culture. Primary cortical culture was prepared as reported previously (Ageta-Ishihara et al., 2009). Transfected hemispheres were digested with bovine trypsin (Sigma-Aldrich) and dissociated mechanically. The dissociated neurons were spotted onto PDL-coated glass bottom dishes at a cell density of $2.0 \times 10^{5}$ neurons/dish for imaging experiments and $5.0 \times 10^{5}$ cells/well for the neurite length assay. Cortical neurons were maintained in MEM supplemented with $10 \%$ fetal bovine serum (Hyclone) and homemade NS-21 supplement (Chen et al., 2008). Half of the medium was replaced every $2 \mathrm{~d}$.

Live imaging. Cortical neurons were labeled at E15.5 using in utero electroporation by injecting pCAG-Lck-G-CaMP7/pCAG-mCherryKRasCT ( $4: 1$ volume ratio) $4 \mu \mathrm{g} / \mu \mathrm{l}$ DNA solution into lateral ventricles. All imaging experiments were performed at $37^{\circ} \mathrm{C}$ in the imaging chamber (INUB-ZILCS-F1; Tokai Hit) under continuous supply of $5 \% \mathrm{CO}_{2}$. Polarized neurons were chosen based on their single long protrusion. Neurons with complex dendritic geometry were excluded from recordings. We also selected cells that expressed the calcium indicator moderately to avoid potential cytotoxicity of the probe and/or poor signal-to-noise ratio. All drugs were added through a syringe attached to the lid of the imaging dish. Images were acquired with an exposure time of $100 \mathrm{~ms}$ and $1 \mathrm{~s}$ intervals in most experiments. Samples were illuminated with the Lambda DG-4 xenon lamp light source (Sutter Instruments) and excitation filters were switched online. The emission filters were controlled mechanically by MAC5000 (Ludl) and synchronized with excitation filter switching. Emitted light was collected with C9100-12 EM-CCD (Hamamatsu Photonics) and all devices were under control of AQUACOSMOS software (Hamamatsu Photonics). The optical system is summarized in Figure $1 C$. Neurons that died during imaging were excluded from further analysis.

Filters, mirrors, and objectives used in this study were as follows: excitation filters: FF01-474/23 (Semrock) for GCaMPs; FF01-575/15 (Semrock) for mCherry; emission filters: FF02-520/28 (Semrock) for GCaMPs, ET630/75m (Chroma) for mCherry; dichroic mirror: 51019bs (Chroma); objective lens: $60 \times$ numerical aperture (NA) 1.49 or $100 \times$ NA 1.40 oil-immersion lens (Olympus).

Image processing. Image processing was performed using Fiji and MetaMorph image processing software (Molecular Devices, RRID: SCR_002368). The acquired $256 \times 256$ pixel image stacks were converted to Tiff format and aligned using the Fiji StackReg plugin if necessary. After subtracting background intensity, we set 6-pixel-wide $(\sim 2 \mu \mathrm{m})$ linear regions of interest (ROIs) so that the neurites of interest remain in the ROIs during the whole imaging period. We used the "kymograph" command in MetaMorph to obtain a 2D "raw kymograph," which had a horizontal spatial axis and a vertical temporal axis.
Briefly, the raw kymograph was obtained as follows:

STEP 1: Time-invariant linear ROIs were set on the acquired stacks. We first obtained a 1D fluorescence intensity profile from the image taken at a given time. Each linear ROI has its own spatial coordinate along its axis and the fluorescence intensity was averaged spatially perpendicular to ROI by 6-pixel-width $(\sim 2 \mu \mathrm{m})$ to reduce potential shot noise. If the length of the ROI is $X_{0}$, then a one (vertical axis: temporal) by $X_{0}$ (horizontal axis: spatial) 1D fluorescence profile is obtained. Multiple ROIs gave the corresponding number of $1 \mathrm{D}$ fluorescence profiles.

STEP 2: We repeated STEP 1 for all acquired frames. If the number of the frames is $T_{0}$, then we repeated STEP $1 T_{0}$ times. We combined the obtained 1D profiles vertically in the temporal order from 1 to $T_{0}$.

STEP 3: For multiple ROIs, we combined the images obtained in STEP 2 spatially (i.e., horizontally). The resultant image was called the raw kymograph.

The obtained 2D raw kymograph was represented as $F_{\text {raw }}(x, t)$, which has two variables, space $(x)$ and time $(t)$. Estimation of "basal intensity" was done by averaging the lower $20 \%$ intensity of $F_{\text {raw }}(x, t)$ during the whole imaging period at each spatial point $x$ and defined as spatial function $F_{0}(x)$. For pharmacological experiments, basal intensity was calculated from the period before drug addition.

All raw kymographs were normalized by the following normalization formula:

$$
F(x, t)=\frac{F_{\text {raw }}(x, t)-F_{0}(x)}{F_{0}(x)}
$$

Fluorescence bleaching was corrected by assuming single exponential decay. The resultant images were simply called kymographs. All processing steps after making raw kymograph were done using Matlab2014a (The MathWorks, RRID:SCR_001622). Kymographs are color coded so that high calcium pixels are represented in warmer colors.

Quantification of calcium elevations. Cumulative calcium elevations (CCEs) and the drug modulation index (DMI) were defined as follows. Given that the spatial length of the kymograph is $X_{0}$ and the imaging period used for obtaining CCE is from $T_{1}$ to $T_{2}$, CCE and DMI are calculated from the following formulas:

$$
\begin{gathered}
\mathrm{CCE}=\frac{\sum_{x=1}^{X_{0}} \sum_{t=T_{1}}^{T_{2}} F(x, t)}{X_{0} \times\left(T_{2}-T_{1}+1\right)} \\
\mathrm{DMI}=\frac{\mathrm{CCE}(10 \text { minutes } \text { after drug addition })}{\operatorname{CCE}(10 \text { minutes } \boldsymbol{b} \text { efore drug addition })}
\end{gathered}
$$

As its definition, CCE is normalized both spatially and temporally and it represents the total amount of calcium influx normalized by time. We were therefore able to compare CCE not only within neurons but also across them. The parameters used in our experiments are $T_{1}=1, T_{2}=$ $1500\left(T_{1}=1, T_{2}=1000\right.$ for experiment shown in Fig. $\left.3 B\right)$. CCEs in drug experiments were separately calculated as follows: $T_{1}=1, T_{2}=600$ for before drug addition, $T_{1}=601, T_{2}=900$ for the early phase, and $T_{1}=$ $901, T_{2}=1500$ for the late phase.

Pharmacological experiments. All drugs were diluted with conditioned culture media and added via syringe. The drugs were purchased from the following manufacturers: diltiazem hydrochloride, and thapsigargin were from Sigma-Aldrich; nimodipine, D-APV, CNQX, and FPL 64176 were from Tocris Bioscience; tetrodotoxin (TTX) was from Sumitomo Dainippon Pharma; EGTA was from Nacalai Tesque; ryanodine was from Abcam; and $\omega$-conotoxin MVIIC was from Peptide Institute. The effects of the drugs on SRCaTs were quantified based on DMI.

CCE comparison between axons and dendrites. For comparison of CCE between axons and dendrites, we set axonal and dendritic ROIs determined by morphology. Axonal and dendritic CCE were calculated for each neuron and the data were pooled. We compared them in cellmatched condition (within cell). The neurites that went out of focus during the imaging period were excluded from further analysis. 
Immunocytochemistry. Cultured neurons were fixed in 4\% paraformaldehyde (PFA)/PBS for $20 \mathrm{~min}$ at room temperature (RT). Fixed samples were blocked in PBS-based blocking solution containing 5\% normal goat serum, $1 \%$ albumin, and $0.2 \%$ saponin for $1 \mathrm{~h}$ at RT. Antibody reactions were performed at $4^{\circ} \mathrm{C}$ overnight. Incubated samples were washed in PBS three times and then incubated in Alexa Fluor-conjugated second antibody solution with Hoechst for $90 \mathrm{~min}$ at RT. The samples were then washed in PBS three times and mounted onto slides.

Perfusion fixation. Animals were anesthetized with lethal dose of pentobarbital and perfused transcardially with PBS followed by $4 \%$ PFA/PBS for 5-10 min. Fixed brain tissue was removed from the skull and postfixed in $4 \% \mathrm{PFA} / \mathrm{PBS}$ at $4^{\circ} \mathrm{C}$ overnight. Fixed brains were transferred to $10 \%, 20 \%, 30 \%$ sucrose/PBS gradually for cryoprotection.

Immunohistochemistry. Transcardially perfused brains were embedded in optimal cutting temperature compound (VWR) and sectioned with HM560 cryostat (Thermo Scientific). Next, $50-\mu$ m-thick brain sections were washed with PBS and blocked with PBS-based blocking solution containing 5\% normal goat serum, $1 \%$ albumin, and $0.3 \%$ Triton $\mathrm{X}-100$ for $2 \mathrm{~h}$ at RT. Antibody reactions were performed at $4^{\circ} \mathrm{C}$ overnight for $3 \mathrm{~d}$. Sections were washed with PBS three times and then incubated in Alexa Fluor-conjugated second antibody solution with Hoechst for 90 min at RT. The sections were further washed in PBS three times and embedded in polyvinylalcohol-based homemade mounting medium. The concentrations of the antibodies were as follows: first antibody 1:500 rabbit polyclonal anti-Ca 1.2 , first antibody 1:500 rabbit polyclonal anti$\mathrm{Ca}_{\mathrm{v}} 1.3$, second antibody 1:500 Alexa Fluor 555 conjugated anti-rabbit IgG for Figure $4 A$; first antibody 1:500 mouse monoclonal anti-GFP, first antibody 1:500 rabbit monoclonal anti-HA, second antibody 1:500 Alexa Fluor 488 conjugated anti-mouse IgG, second antibody 1:500 Alexa Fluor 647 conjugated anti-rabbit IgG for Figure $4 B$; first antibody 1:500 rabbit monoclonal anti-HA, second antibody 1:500 Alexa Fluor 647 conjugated anti-rabbit IgG for Figure 4C; first antibody 1:500 rabbit polyclonal antiGFP, first antibody 1:500 rat monoclonal anti-mCherry, second antibody 1:250 Alexa Fluor 488 conjugated anti-rabbit IgG for GFP, second antibody 1:250 Alexa Fluor 594 conjugated anti-rat IgG for Figure 6, $B$ and $C$; first antibody 1:2000 rabbit polyclonal anti-GFP, second antibody 1:500 Alexa Fluor 488 conjugated anti-rabbit IgG for Figure 7.

Antibodies. Primary antibodies used in this study were as follows: rabbit polyclonal anti-GFP (Thermo Fisher Scientific, A11122, RRID: AB_221569), rat monoclonal anti-GFP (Nacalai, GF090R, RRID: AB_10013361), mouse monoclonal anti-GFP (Thermo Scientific, 3E6, RRID:AB_2313858) rabbit polyclonal anti-DsRed (Clontech, 632496, RRID:AB_10013483), rat monoclonal anti-mCherry (Thermo Scientific, M11217, RRID:AB_2536611), rabbit polyclonal anti-Ca 1.2 (Alomone Laboratories, ACC-003, RRID:AB_2039771), rabbit polyclonal anti$\mathrm{Ca}_{\mathrm{v}} 1.3$ (Alomone Laboratories, ACC-005, RRID:AB_2039775), mouse monoclonal anti-HA (Cell Signaling Technology, 6E2, RRID: AB_2314619), rat monoclonal anti-HA (Roche, 3F10, RRID: AB_2314622), rabbit monoclonal anti-HA (Cell Signaling Technology, C29F4, RRID:AB_1549585), rabbit polyclonal anti-GluR1 (Millipore, 07-660). Secondary antibodies used were as follows: Alexa Fluor 488-, 555-, 594-, and 647-conjugated anti-mouse/rat/rabbit IgG antibodies (Life Technologies).

Western blotting. Cortical neuronal culture was prepared from postnatal day 0 (P0) pups of homozygous floxed Cacnalc mice. After 1 week, $1.5 \times 10^{6}$ cortical neurons were infected with $3.0 \mu \mathrm{l}$ of viral solution $\left(1.1 \times 10^{13} \mathrm{gc} / \mathrm{ml}\right)$ of an AAV-expressing iCre-GFP plasmid (kindly provided by Thomas Sudhof). Noninfected neurons were used as a control. GFP fluorescence was observed in most cells within $5 \mathrm{~d}$. Cells were lysed at 2 weeks after infection. Neurons were collected with cell scrapers and sonicated in sucrose-based solution containing the following (in mM): 250 sucrose, 10 Tris, 10 HEPES, and 1 EDTA, pH 7.2 with $\mathrm{NaOH}$. To prevent protein degradation, the Complete-Mini proteinase inhibitor mixture (Roche Diagnostics) was added to the solution.

Cell homogenates were centrifuged at $4000 \times g$ for $10 \mathrm{~min}$ at $4^{\circ} \mathrm{C}$ and the supernatant was further centrifuged at $50000 \times g$ for $1 \mathrm{~h}$ at $4^{\circ} \mathrm{C}$ to enrich membrane fraction. Centrifuged pellets were resuspended in the sucrose-based solution and protein concentration was determined with the BCA Protein Assay Kit (Thermo Scientific). Proteins were denatured in Laemmli buffer supplemented with 5\% 2-mercapto-ethanol. The protein was denatured for $10 \mathrm{~min}$ at $70^{\circ} \mathrm{C}$. Proteins $(10$ or $20 \mu \mathrm{g} / \mathrm{lane}$ depending on the width of the lanes) were separated in $7 \%$ SDSpolyacrylamide gel. Protein bands were transferred onto polyvinylidenedifluoride membranes ( $0.45 \mu \mathrm{m}$ pore size; Merck Millipore) in methanol-free transfer solution supplemented with $\operatorname{SDS}\left(30 \mathrm{~V}, 4^{\circ} \mathrm{C}\right.$, overnight $)$.

The membranes were blocked in blocking buffer (PBS containing 5\% skimmed milk, $1 \%$ albumin, and $0.05 \%$ Tween 20 ) for $2 \mathrm{~h}$ at RT. The blocked membrane was incubated in 1:1000 first antibody containing 1/2 blocking buffer (PBS containing 2.5\% skimmed milk, $0.5 \%$ albumin, and $0.05 \%$ Tween 20 ) overnight at $4^{\circ} \mathrm{C}$. GluR1 protein was used as a loading control for membrane protein. The incubated membranes were washed in PBS $/ 0.05 \%$ Tween 20 three times each for $5 \mathrm{~min}$ and incubated in 1:5000 second antibody (HRP-conjugated anti-IgG) containing $1 / 2$ blocking buffer for $90 \mathrm{~min}$ at RT. The membranes were washed in PBS/ $0.05 \%$ Tween 20 three times each for $5 \mathrm{~min}$.

The HRP-positive bands were detected using ECL select (GE Healthcare Life Sciences) and chemiluminescence images were acquired by LAS-4000 Mini (Fujifilm). The obtained images were analyzed using ImageJ.

Pharmacological knock-out experiment. A DHP-insensitive calcium channel (pCAG-sHA-Ca $1.2-\mathrm{T} 1039$ Y or its mutant) with pCAG-Ca $\alpha 2 \delta 1$ T2A-Ca $\beta 3$-P2A-mCherry-KRasCT auxiliary subunits and pCAG-Lck-GCaMP7 1:1:2 mixture was introduced into cortical neurons using in utero electroporation at E15.5. Then, $10 \mu \mathrm{M}$ nimodipine was added into the imaging medium to isolate the DHP-insensitive fraction of SRCaTs.

Quantification of neurite length. Future layer 2/3 excitatory cortical neurons were labeled by injecting $4 \mu \mathrm{g} / \mu \mathrm{l}$ pCAG-EGFP-KRasCT or pCAG-Cre-P2A-mCherry-KRasCT at E15.5 as described in the "In utero electroporation" section. Drugs were added to the culture media $6 \mathrm{~h}$ after plating if necessary. Neurons were fixed 48 or $72 \mathrm{~h}$ after plating depending on the experiment. Fixed neurons were immunoenhanced against fluorescent proteins to facilitate tracing of neurites. Images were acquired using a DP70 microscope (Olympus). Axons and dendrites were determined by morphology as described previously (Ageta-Ishihara et al., 2009). The longest thin neurite was defined as an axon. The lengths of the neurites were quantified using the NeuronJ plugin (Meijering et al., 2004). All axonal or dendritic tracings were summed according to the neurite type and subjected to analysis. For the $\mathrm{Ca}_{\mathrm{v}} 1.2$ conditional knock-out experiment, the plasmids were electroporated at E12.5 to minimize the effect of residual calcium channel. Control neurons and Cre-expressing neurons were seeded on the same coverslip to reduce cell density effect on neurite length. Outliers with a neurite length longer or shorter than the average by 3 SDs were excluded; such cells were very few.

Quantification of ectopic cells. The transfection marker pCAG-mEGFP and other plasmids (calcium channels, auxiliary subunits, and TRE inducer for doxycycline experiment at total concentration of $4 \mu \mathrm{g} / \mu \mathrm{l}$ ) were electroporated into ICR mice embryos at E15.5. The ratio of plasmid was different from experiment to experiment. The ratio of pCAG-EGFP/ pCAG-Ca 1.2 or its variants/auxiliary subunits was $1: 1: 1$ for constitutive expression experiment and the ratio of pCAG-EGFP/TRE-Ca 1.2 or its variants/auxiliary subunits/pCAG-tTA or rtTA was 2:2:2:1 for doxycycline experiments. Pups were raised and perfused transcardially at P16. After immunostaining, fluorescence images were acquired with an LSM 510 microscope (Zeiss). Neurons below layer 2/3 were counted and the location was confirmed according to Hoechst image. The most superficial nuclei-rich layer was defined as layer $2 / 3$ and the border to the deeper layer was considered as its lower edge. The ectopic cell density was calculated as the number of ectopic cells divided by ROI area. The migration process can be affected by the exact date of in utero electroporation. Hours after fertilization can slightly vary from animal to animal. To minimize such variations, we injected control and gene of interest into both sides of the uterus and set this as the internal control.

Postnatal gene expression/suppression by doxycycline. Doxycycline was administered postnatally by replacing the mother's drinking water with $1 \%$ doxycycline $10 \%$ sucrose containing water at P1. Doxycycline was delivered to pups through mother's milk. 
Characterization of SRCaTs mediated by different calcium channels. Cells were prepared as in pharmacological knock-out experiments. All neurons were imaged in the presence of $10 \mu \mathrm{M}$ nimodipine. SRCaT events were identified manually on kymographs and their parameters analyzed using Fiji imaging software. For each SRCaT event, a 2D ROI was selected on the kymograph. The ROI width on the $x$-axis and the ROI height on the $y$-axis were defined as spatial spread and duration, respectively. The average fluorescence intensity associated with this 2D ROI was defined as SRCaT amplitude.

$q R T$-PCR of neonatal RNA expression of L-type calcium channels. Brains were obtained from ICR mice pups every $24 \mathrm{~h}$ from P0 onwards. On the last day of the experiment (P4), a control adult brain was obtained from the mother. Animals were decapitated and brains were removed from the skull and frozen in liquid nitrogen immediately. RNA was extracted by TRIzol (Thermo Scientific) and purified with the RNeasy kit (Qiagen). Crude RNA was further purified and digested with DNaseI for $1 \mathrm{~h}$ at RT. DNase-treated RNA was reverse-transcribed using the Transcriptor kit (Roche). qRT-PCR was performed using SYBR Premix Ex Taq (Tli RNase H Plus; TaKaRa). The primers were designed using the Universal ProbeLibrary website https://lifescience.roche.com/global_en/brands/ universal-probe-library.html. The level of RNA expression was normalized by GAPDH. The primers used in this study were as follows: GAPDH-F 5'-TGTCCGTCGTGGATCTGAC-3', GAPDH-R 5'-CCTG CTTCACCACCTTCTTG-3'， Ca 1 1.2-F 5'-TAGTGTCCGGAGTCCC AAGT-3', Ca 1 1.2-R 5'-GAAGAGCACAAGAAGGGCAAT-3', Ca 1 1.3-F 5' -GAAGCTGCTTGACCAAGTTGT-3', Ca 1 1.3-R 5' -AACTTCCCCAC GGTTACCTC-3'.

Subcellularlocalization of calcium channels.pCAG-EGFP/pCAG-Ca $\mathrm{a}_{\mathrm{v}} \alpha 2 \delta 1-$ T2A-Ca $\beta$ ק3-P2A-mCherry-KRasCT/pCAG-sHA-Ca $1.2-$ G406R-T1033Y mixture solution was transfected into E15.5 ICR mice embryos by in utero electroporation at a ratio of $1: 1: 1$. Pups were perfused transcardially at P1 and P3. The subcellular localization of calcium channels were determined by Alexa Fluor-647 immunostaining against EGFP and HA antigen. To examine endogenous subcellular localization of calcium channels, we used the SLENDR method (Mikuni et al., 2016). pX330U6-Chimeric_BB-CBh-hSpCas9-Ca 1 1.2-gRNA plasmid and pCAGmEGFP-KRasCT $\left(1 \mu \mathrm{g} / \mu \mathrm{l}\right.$ each) and ssODNs against $\mathrm{Ca}_{\mathrm{v}} 1.2(20 \mu \mathrm{M})$ were transfected by in utero electroporation at E12.5. Pups were transcardially perfused postnatally and the localization of endogenous channel was determined by anti-HA immunohistochemistry. The sequences designed to target Cav1.2 were identical to those used in Mikuni et al. (2016).

Experimental design and statistical analysis. All statistical values are provided in the legend of the corresponding figures. Here, we summarize the experimental design of each experiment briefly. Calcium imaging data of 38 neurons in Figure 2 were obtained from six independent batches. The obtained data were pooled and ROIs were set manually. CCE in dendrites and that in axons were compared with Wilcoxon matched pair test $(n=$ 38 neurons, one neuron from one dish, pooled data).

To evaluate the effect of membrane hyperpolarization on SRCaTs, we used Mann-Whitney test in Figure $3 B(n=12$, pooled data from two independent experiments). The data of pharmacological experiments described in Figures 3 and 4 and Table 1 were collected from at least three independent experiments except for the EGTA, ryanodine, and diltiazem experiments. We initially screened all drug effects based on DMI with a sample size of $\sim 10$ cells (one cell per dish) per condition, using onesample $t$ test against one as a criterion. We then proceeded to a further quantification round for the $\mathrm{Ca}^{2+}$ channel drugs. The obtained CCE data were normalized as described in the "Quantification of calcium elevations" section. Similarly, for the pharmacological knock-out experiment, we used a one-sample $t$ test against one. In Figure 5, we pooled data from five cells in each condition.

To evaluate the effect of genotype and drug on neurite length, we used a two-way ANOVA in Figure $6 \mathrm{~B}$. We pooled data from two independent experiments. Because we found both factors to be significant, we proceeded to post hoc analysis. The effect of genotype was compared with Mann-Whitney test and the effect of drug was analyzed with Kruskal-Wallis test followed by Dunn's multiplecomparisons test. The effect of Cre expression on neurite length was compared with Mann-Whitney test. Data in Figure 7, B-E, were obtained from at least two individual experiments. To quantify radial migration, we measured ectopic cell density from one slice per pup. We pooled ectopic cell density data from at least two mothers and compared them with an unpaired $t$ test. All statistical analyses were performed using Prism 7 (GraphPad) software.

\section{Results \\ Submembraneous calcium imaging identifies frequent SRCaTs, especially in developing cortical axons}

To record calcium influxes that are generated spontaneously in developing cortical neurons, we expressed a membrane-anchored GECI, Lck-G-CaMP7, through in utero electroporation and performed time-lapse imaging. In utero delivery at E15.5, $3 \mathrm{~d}$ before cortical dissection and plating, enabled us to restrict the ultimate expression cell type to the excitatory cortical neurons of layer $2 / 3$ and to obtain a high expression of the probe at the time of plating (Fig. 1A-C). Lck-G-CaMP7-expressing neurons, imaged at days in vitro (DIV) 0-2 revealed strikingly robust spontaneous calcium elevations that were generated both in axons and in dendrites (Fig. 1D, Movie 1). Spontaneous calcium elevations were often localized and relatively slow events ( $\sim 10 \mathrm{~s}$ duration) (Fig. $1 D$, bottom), sometimes even in the absence of detectable somatic calcium rises, and were visible only hours after the nascence of the very precocious neurites (Fig. $1 E$ ). These spatiotemporal characteristics were clearly distinct from the calcium elevations exerted by action potentials or synaptic transmission (Inoue et al., 2015). We thus termed these events SRCaTs, which were evident as early as 4 or $6 \mathrm{~h}$ after plating, when the neuronal polarity had not yet been clearly determined (Movies 2, 3). SRCaTs were observed throughout the period of polarity formation and subsequent growth of axons and dendrites. Some SRCaTs were confined to a single neurite, whereas others spread over the whole cell.

To test whether SRCaTs were differentially regulated between axons and dendrites, we performed a kymograph analysis along the neurite axis and recorded SRCaTs' spatiotemporal dynamics (Wang and Schwarz, 2009). Based on a 1D stack of a ROI that was chosen on a neurite, time profiles of fluorescence stacks were subsequently obtained and then normalized and color coded to visualize SRCaT profiles in 3D (Fig. $2 A, B$ ). Kymographs showed that Lck-G-CaMP7 enabled us to unambiguously detect subtle fluorescence changes even in fine neurites with much higher spatial contrast than using cytosolic G-CaMP7, probably due to higher surface to volume ratio. Other Lck peptide-fused GECIs, such as Lck-GCaMP6s or Lck-GCaMP6m, did not show significant improvement over Lck-G-CaMP7 (data not shown), but confirmed that SRCaTs were robust events. Because it is reported that GCaMP6m and GCaMP6s have higher affinity and slower calcium dissociation kinetics than G-CaMP7, our results were consistent with the idea that the characteristics of G-CaMP7 might indeed be suitable for detecting calcium changes with high temporal resolution. Some calcium elevations were apparently repetitive/oscillatory and spreading in space (Fig. 2C, left), which implied that some SRCaTs might be regenerated locally, as opposed to others that subsided after one event (Fig. $2 C$, right). We found little evidence for a unitary calcium elevation because the putative smallest calcium elevations that we recorded greatly varied both in amplitude and duration within our detection limits. The rise time (several to $10 \mathrm{~s}$ ) of the intracellular calcium concentration was faster than its decaying speed. Sometimes, spontaneous calcium elevations propagated from the source along neurites to adjacent regions (Fig. 1E). 
A

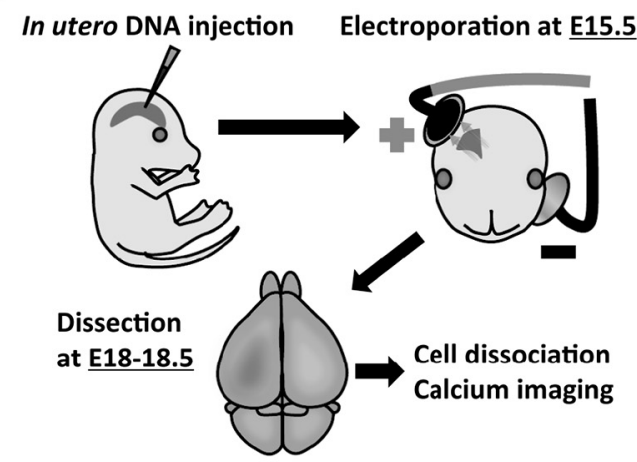

B

\begin{tabular}{|l|l|l|l|l|}
\hline Lck & M13 & EGFP-C-half & EGFP-N-half & CaM (2-148) \\
\hline
\end{tabular}
C

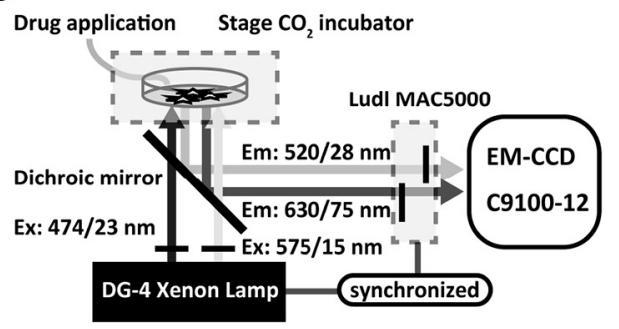

D
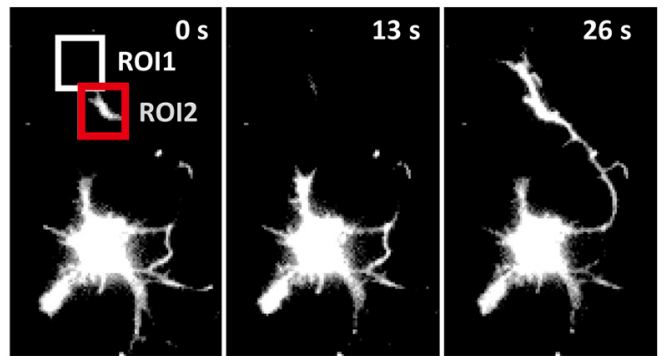

Time $0 \mathrm{~s}$

$10 \mathrm{~s}$

$20 s$

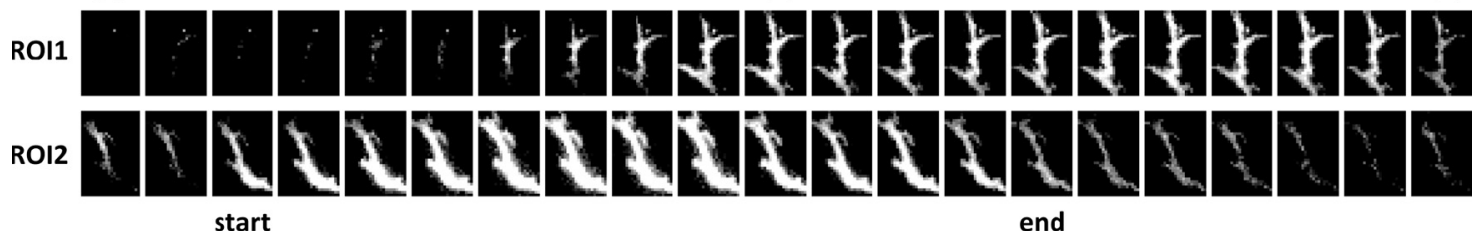

E

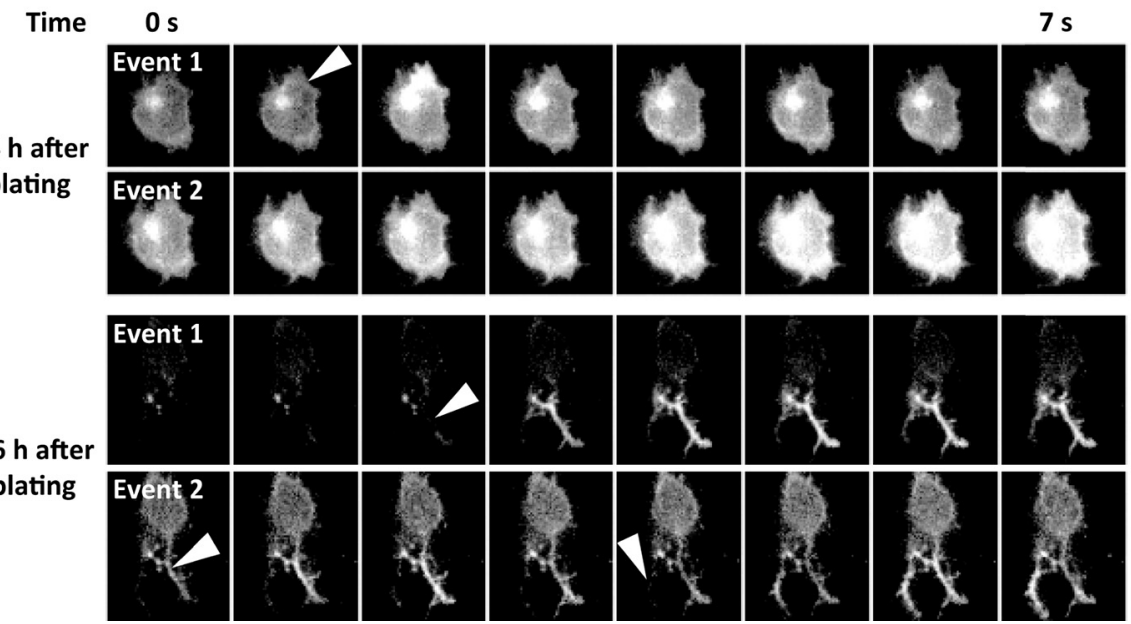

Figure 1. Calcium imaging of developing neurons using membrane-tethered G-CaMP7. A, Selective labeling of future layer 2/3 excitatory cortical neurons by in utero electroporation at E15.5. Injection of Lck-G-CaMP7-expressing plasmids, in utero electroporation, dissociation, plating, and recording of electroporated neurons at E18 to E18.5. Neurons labeled at E15.5 are known to develop into layer 2/3 excitatory cortical neurons. B, Primary structure of Lck-G-CaMP7. Membrane targeting sequence from human tyrosine kinase Lck was fused to the N terminus of G-CaMP7. Lck-G-CaMP7 was irreversibly inserted to plasma membrane by posttranslational lipid modification. C, Live imaging microscopy system. Lck-G-CaMP7-expressing neurons were imaged in culture medium at $37^{\circ} \mathrm{C}$ under continuous supply of $5 \% \mathrm{CO}_{2}$. Neurons were illuminated with DG-4 xenon lamp and excitation and emission filters were mechanically switched. Fluorescence was detected by $\mathrm{C} 9100-12$ EM-CCD camera. Drugs were added through the syringe attached to the lid of the imaging dish. D, Representative fluorescence images of DIV 2 Lck-G-CaMP7-expressing neurons. Top, Whole-cell view (13 s intervals). Scale bar, $10 \mu \mathrm{m}$. Spontaneous calcium elevations were observed in the middle of the axon. Two ROls are shown in red and white. Bottom, Magnified view from each R0I (1 s intervals). Note that calcium elevations are slow and localized. Onset and offset of spontaneous calcium elevations are shown. $\boldsymbol{E}$, Representative fluorescence images of Lck-G-CaMP7-expressing neurons $4 \mathrm{~h}$ (top) and $6 \mathrm{~h}$ (bottom) after plating. Most calcium elevations were localized (arrowheads), whereas whole-cell calcium elevation also existed (second row). Some calcium elevations involved adjacent neurites (arrowheads, bottom). Images were acquired at 1 s intervals. Scale bars, $5 \mu \mathrm{m}$.

We next investigated SRCaTs in axons and in dendrites. Both peak amplitudes and cumulative integrals of "timeaveraged areas under the curve" (which we here call CCEs for simplicity) were significantly higher in axons than that in the dendrites of the same cells (dendrites, CCE $=0.50 \pm 0.26$
$/ \mu \mathrm{m} / \mathrm{s}, n=38 ;$ axons, $\mathrm{CCE}=0.74 \pm 0.48 / \mu \mathrm{m} / \mathrm{s}, n=38 ; \mathrm{W}=$ $-478, p=0.0003$, Wilcoxon matched pair test, mean $\pm \mathrm{SD}$ ). A similar result was obtained when examining peak CCE (CCE index from the pixels with $\Delta F / F_{0}$ that ranked in the top $5 \%$ at each spatial point during the whole imaging period; dendrites, 


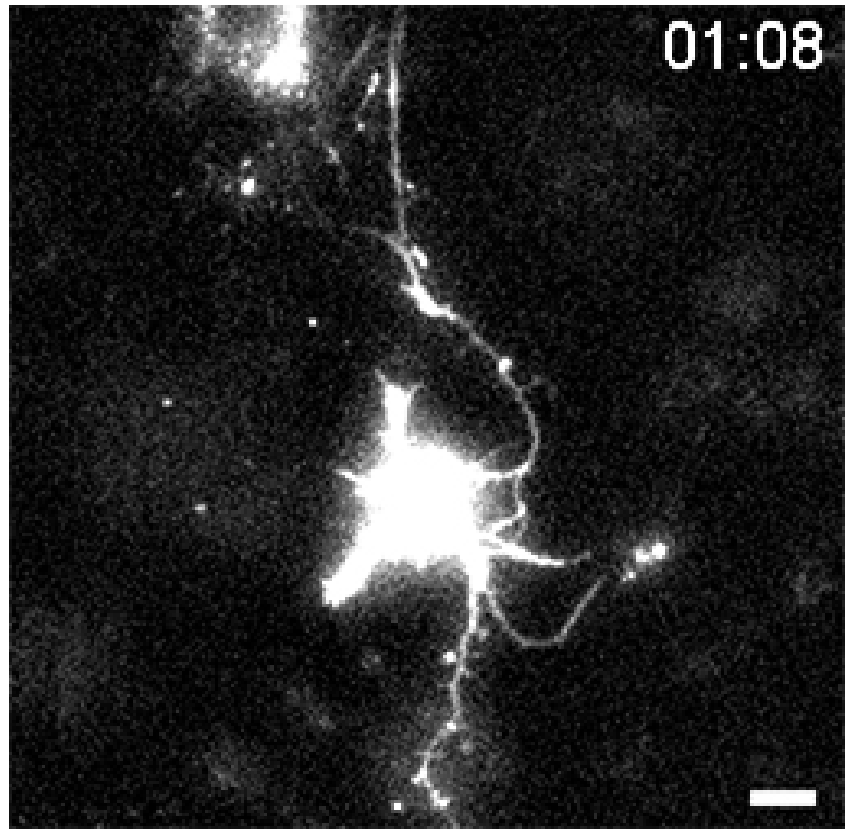

Movie 1. Spontaneous calcium elevations in a DIV 2 neuron. This is a representative movie obtained from a DIV 2 Lck-G-CaMP7-expressing cortical neuron. SRCaTs are evident in neurites. Images were acquired every $1 \mathrm{~s}$ at 25 frames $/ \mathrm{s}$. Scale bar, $5 \mu \mathrm{m}$.

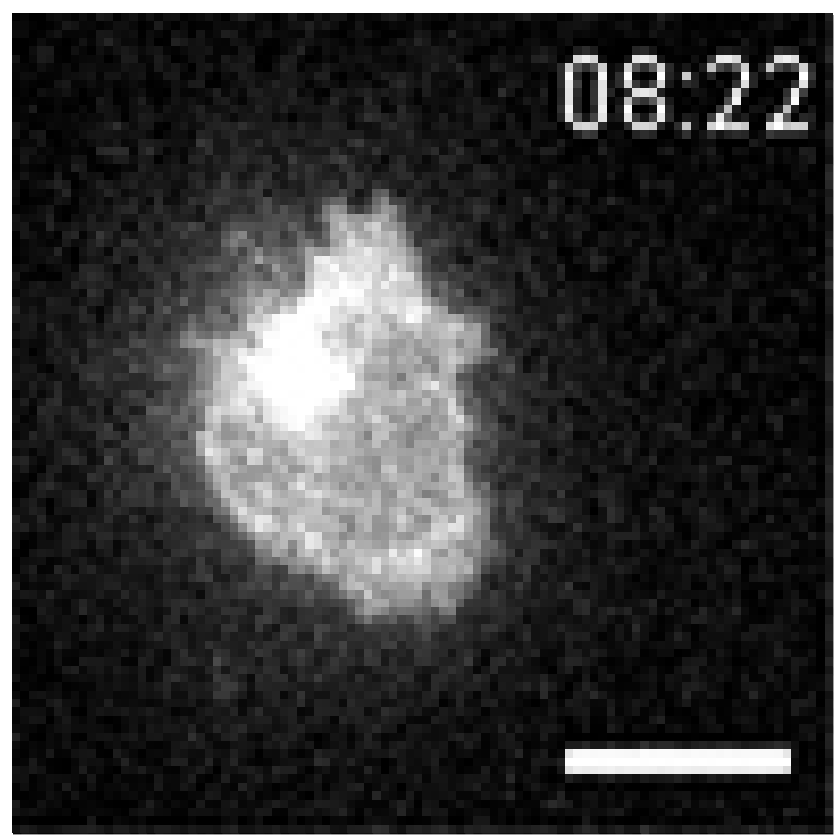

Movie 2. Spontaneous calcium elevations $4 \mathrm{~h}$ after plating. This is a representative movie obtained from an Lck-G-CaMP7-expressing cortical neuron $4 \mathrm{~h}$ after plating. Images were acquired every $1 \mathrm{~s}$ at 25 frames/s. Scale bar, $10 \mu \mathrm{m}$

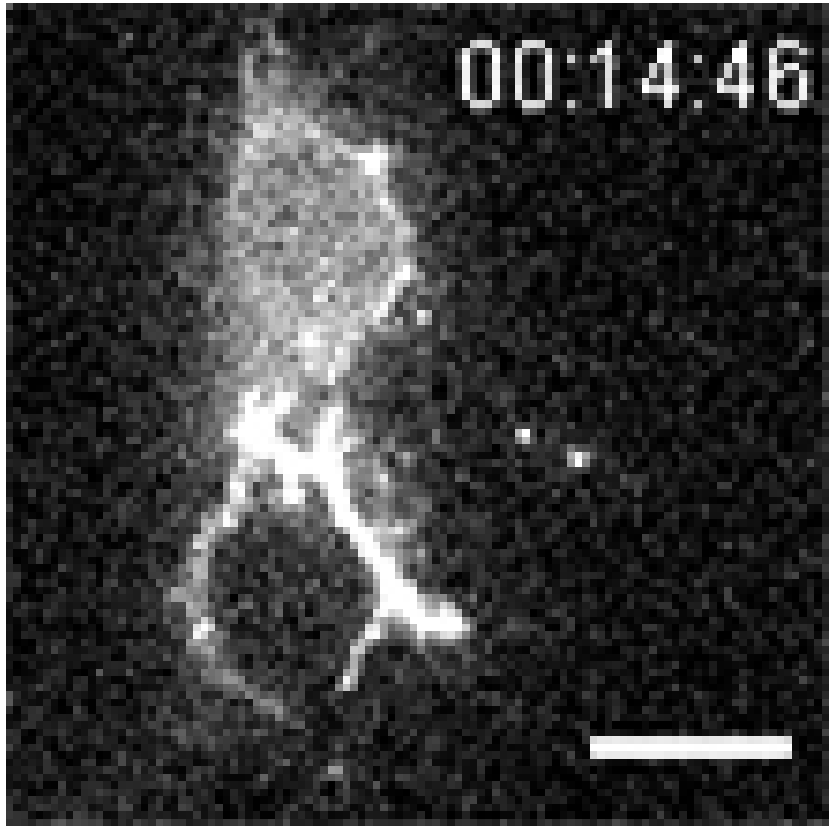

Movie 3. Spontaneous calcium elevations $6 \mathrm{~h}$ after plating. This is a $\square$ representative movie obtained from Lck-G-CaMP7-expressing cortical neuron $6 \mathrm{~h}$ after plating. Images were acquired every $1 \mathrm{~s}$ at 25 frames/s. Scale bar, $10 \mu \mathrm{m}$.

\section{SRCaTs are primarily driven via L-type VGCCs}

We next investigated what calcium sources most contributed to SRCaTs. We performed pharmacological experiments to screen the possible candidates. We measured CCE from Lck-G-CaMP7expressing DIV 1-2 neurons before and after drug addition and calculated a DMI that would be significantly larger or smaller than 1 when the drug enhances or diminishes SRCaTs, respectively. The drug effect was evaluated in the early ( $0-5$ min after drug addition) and the late phase (5-15 min after drug addition) because some drugs needed several minutes to act. First, we decreased the calcium concentration of the surrounding media by adding EGTA. When the extracellular calcium was chelated by EGTA, almost all SRCaTs were abolished, as summarized in Table 1 . Therefore, the primary source of SRCaTs was found to be extracellular calcium.

We first confirmed whether membrane depolarization is required for calcium entry because VGCCs are one of the candidate molecules. To address this possibility, we hyperpolarized membrane voltage by overexpression of an inward rectifying potassium channel $\mathrm{K}_{\mathrm{ir}}$ 2.1. Expression of $\mathrm{K}_{\mathrm{ir}} 2.1$, but not of its hyperpolarizationdeficient point mutant $\mathrm{V} 302 \mathrm{M}$, hyperpolarized membrane potentials (Bendahhou et al., 2003; Ma et al., 2007), thus resulting in significant reduction in SRCaTs in $\mathrm{K}_{\mathrm{ir}}$ 2.1-WT-expressing neurons compared with the V302M-mutant-expressing condition (see Fig. $3 A, B)$. These results suggested that membrane depolarization was critical for generating SRCaTs.

We next tested the effect of a blocker of voltage-gated sodium channels, TTX, at a concentration that blocked action potential generation $(1 \mu \mathrm{M})$ but had no effect on SRCaTs in the early and the late phase (Fig. 3C, D, Table 1). Furthermore, $20 \mu \mathrm{M} \omega$-conotoxin MVIIC (CTX-MVIIC), a broad blocker of N, P/Q-type VGCCs, also showed no effect (Table 1). This result suggested that N, P/Q-type high-threshold VGCCs were not involved in generating SRCaTs. However, $10 \mu \mathrm{M}$ nimodipine, a blocker of voltage-dependent L-type
$\mathrm{CCE}=1.72 \pm 1.09 / \mu \mathrm{m} / \mathrm{s}, n=38$; axons, $\mathrm{CCE}=2.71 \pm 0.30$ $/ \mu \mathrm{m} / \mathrm{s}, n=38 ; \mathrm{W}=499, p=0.0002$, Wilcoxon matched pair test, mean $\pm \mathrm{SD})$, thus ruling out artifacts due to saturation and thresholding.

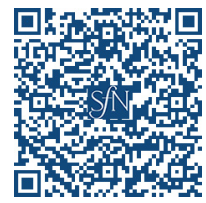


A

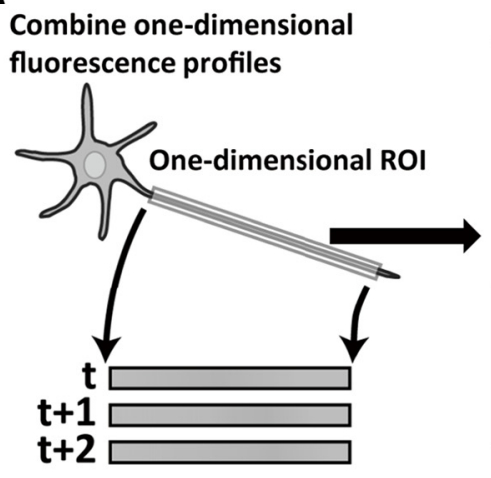

B Set ROIs along neurites ROI1: dendrite, ROI2: axon

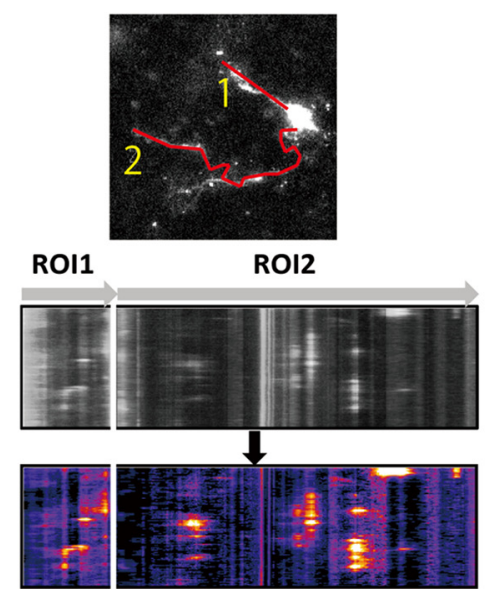

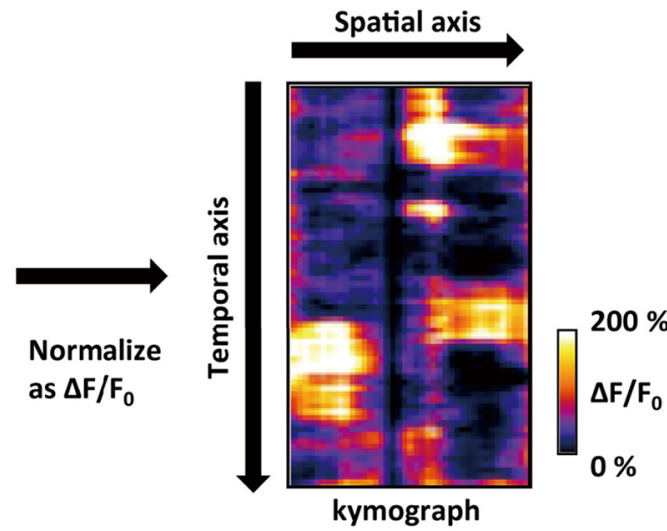

$5 \mu \mathrm{m}$

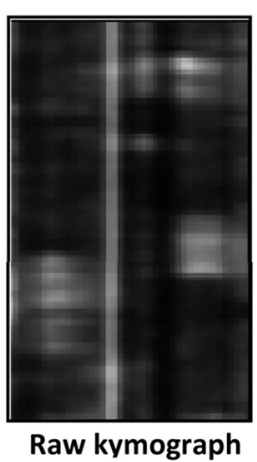

C

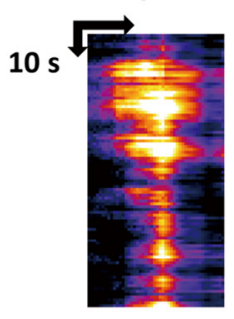

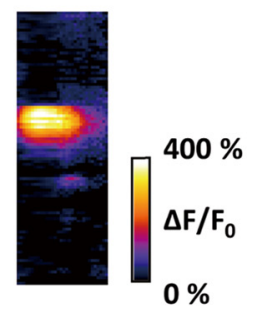

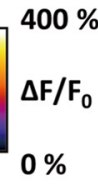

Figure 2. Kymograph-based analysis reveals the highly dynamic nature of SRCaTs. A, Summary of kymograph-based analysis. Left, 1D ROIs were set along neurites and the fluorescence intensity profiles in ROls were obtained from all imaging frames. The obtained 1D fluorescence profiles were combined in the temporal order. Middle, Vertically combined 1D fluorescence profiles (i.e., raw kymograph) were divided by basal intensity profile. Basal intensity profile is defined as the mean of lower $20 \%$ fluorescence intensity at each spatial point during the imaging period. Normalization formula is $F(x, t)=\left\{F_{\text {raw }}(x, t)-F_{0}(\boldsymbol{x})\right\} / F_{0}(\boldsymbol{x})$, where $F_{\text {raw }}(x, t)$ is the raw kymograph and $F_{0}(\boldsymbol{x})$ is the basal intensity profile. Right, 0 btained $2 \mathrm{D}$ images are simply called kymographs. The vertical axis is temporal and the horizontal axis is spatial. $\boldsymbol{B}$, Obtaining kymographs. Example ROIs were set along neurites as shown (left). Raw kymographs were divided by basal intensity profile. The directions of arrows are proximal to distal. C, Representative magnified kymographs of oscillatory pattern (left) and transient pattern (right). Note that calcium concentration rises to the maximum level immediately after the onset (right). SRCaTs are not qualitatively uniform, which implies that there are multiple coexisting SRCaT-generating/maintaining mechanisms.

Table 1. Summary of pharmacological effects on SRCaTs tested in the screening round

\begin{tabular}{|c|c|c|c|c|c|c|c|c|}
\hline Drug name & $\begin{array}{l}\text { Concentration } \\
(\mu \mathrm{M})\end{array}$ & $n$ & $\begin{array}{l}\text { Early } \\
\text { DMI (\%) }\end{array}$ & $\begin{array}{l}\text { SD } \\
(\%)\end{array}$ & $p$ & $\begin{array}{l}\text { Late } \\
\text { DMI (\%) }\end{array}$ & $\begin{array}{l}\text { SD } \\
(\%)\end{array}$ & I \\
\hline TTX & 1 & 14 & 128 & 52 & 0.0625 & 192 & 169 & 0.0621 \\
\hline Nimodipine & 10 & 25 & 56 & 40 & $<0.0001$ & 48 & 47 & $<0.0001$ \\
\hline FPL 64176 & 25 & 16 & 178 & 146 & 0.0478 & 158 & 103 & 0.0396 \\
\hline Diltiazem & 200 & 7 & 21 & 23 & 0.0001 & 7.2 & 16 & $<0.0001$ \\
\hline Thapsigargin & 10 & 12 & 118 & 115 & 0.6006 & 69 & 57 & 0.0866 \\
\hline Ryanodine & 20 & 6 & 119 & 49 & 0.3822 & 125 & 66 & 0.3965 \\
\hline $\begin{array}{c}\omega \text {-Conotoxin } \\
\text { MVIIC }\end{array}$ & 20 & 16 & 142 & 56 & 0.0098 & 144 & 118 & 0.1576 \\
\hline CNQX & 10 & 11 & 108 & 63 & 0.6820 & 110 & 71 & 0.6641 \\
\hline D-APV & 50 & 9 & 167 & 81 & 0.0385 & 147 & 76 & 0.1026 \\
\hline EGTA & 2000 & 4 & 3.0 & 1.6 & $<0.0001$ & 0.2 & 0.3 & $<0.0001$ \\
\hline
\end{tabular}

Values in bold reached statistical significance $(p<0.05)$.

calcium channels significantly diminished SRCaTs (Fig. 3E, left, F, Movie 4). Similar results were obtained using diltiazem, another blocker of L-type VGCCs (Table 1). Together, our results are consistent with the idea that opening of L-type VGCCs was necessary for SRCaT generation, but that $\mathrm{Na}^{+}$spikes were dispensable.
To further test this, we applied $25 \mu \mathrm{M}$ FPL 64176, a potent activator of L-type VGCCs, and found that this was sufficient to increase axonal SRCaTs significantly, with little correlation with somatic calcium transients (Fig. 3E, right, F, Movie 5). CNQX (10 $\mu \mathrm{M})$, a blocker of AMPAR, and D-APV $(50 \mu \mathrm{M})$, a blocker of NMDAR, had no effect on SRCaTs (Table 1). Furthermore, thapsigargin $(10 \mu \mathrm{M})$, a blocker of SERCA that is required for regenerating $\mathrm{IP}_{3}$-dependent $\mathrm{Ca}^{2+}$ stores, and ryanodine $(20 \mu \mathrm{M})$, a blocker of ryanodine receptor that is involved in regulating $\mathrm{Ca}^{2+}$-induced $\mathrm{Ca}^{2+}$ stores, also had no significant effects on SRCaTs (Table 1). However, although the effect of thapsigargin was not significant, a subpopulation of neurons did show lower DMI in the late phase (data not shown), suggesting that intracellular $\mathrm{Ca}^{2+}$ stores may be involved in some but not all SRCaTs.

This pharmacological evidence strongly suggested that spontaneous opening of L-type VGCCs triggered SRCaTs in developing neurons.

Perinatally expressed $\mathrm{Ca}_{\mathrm{v}} 1.2$ and $\mathrm{Ca}_{\mathrm{v}} 1.3$ mediate SRCaTs Our observations that VGCCs may mediate SRCaTs in immature neurons are in sharp contrast with prior reports in adult tissues, in which the predominant pore-forming $\alpha 1$ subunits of L-type 
A

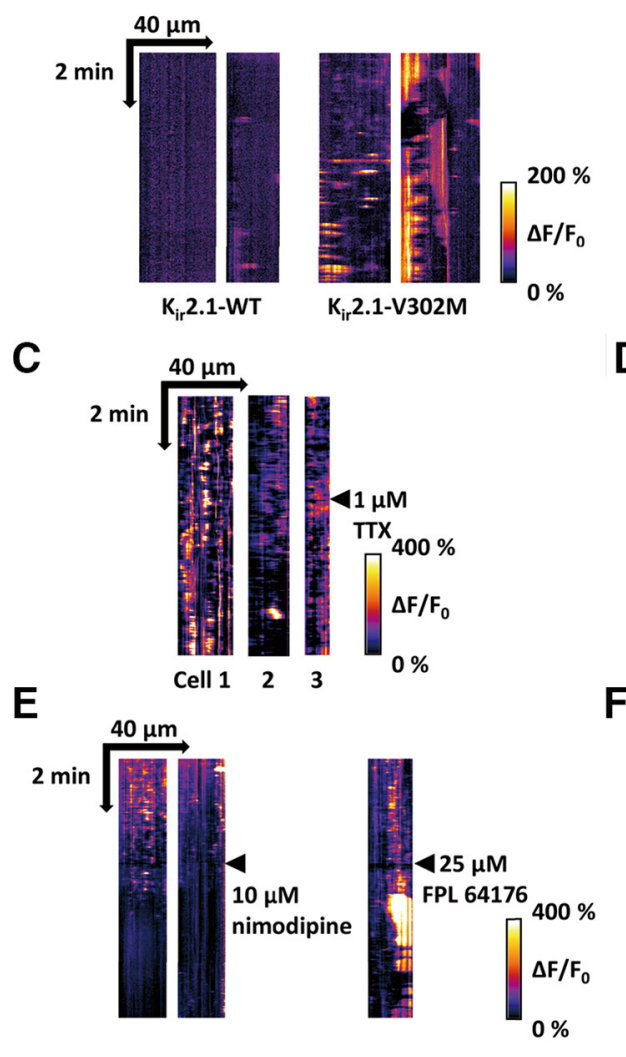

B

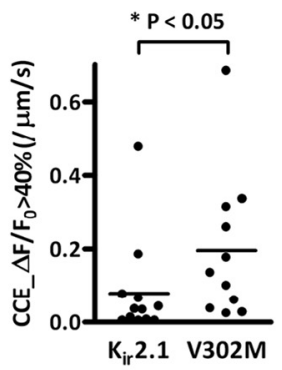

D
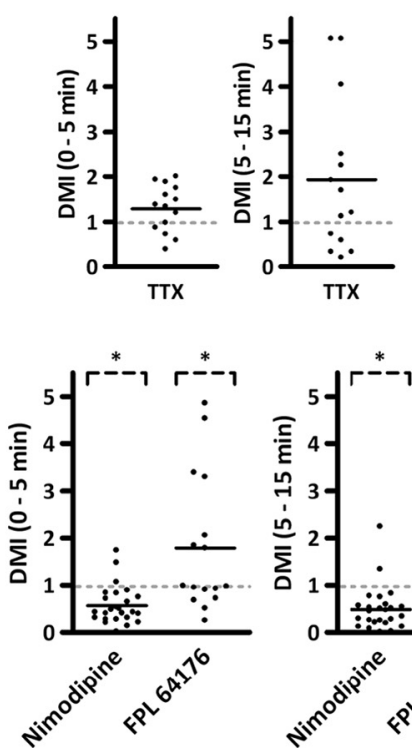

Figure 3. L-type VGCCs mediate SRCaTs. A, Representative kymographs of $\mathrm{K}_{\mathrm{ir}} 2.1$-WT-expressing (left) or $\mathrm{K}_{\mathrm{ir}} 2.1-\mathrm{V} 302 \mathrm{M}$ expressing neurons (right). Less SRCaTs were observed in $\mathrm{K}_{\mathrm{ir}}$ 2.1-WT-expressing neurons than in hyperpolarization-deficient $\mathrm{K}_{\mathrm{i} r} 2.1$ V302M-mutant-expressing neurons. $B$, Comparison of CCE calculated from $\Delta F / F_{0}>40 \%$ pixels $\left(K_{i r} 2.1-\right.$ WT, $C C E \_\Delta F / F_{0}>40 \%=$ $0.08 \pm 0.13 / \mu \mathrm{m} / \mathrm{s}, n=12 ; \mathrm{K}_{\mathrm{ir}} 2.1-\mathrm{V} 302 \mathrm{M}, \mathrm{CCE} \_\Delta \mathrm{F} / \mathrm{F}_{0}>40 \%=0.19 \pm 0.20 / \mu \mathrm{m} / \mathrm{s}, n=12 ;$ Mann-Whitney $U=32.00, p=$ 0.0393 , Mann-Whitney test, mean \pm SD). Membrane hyperpolarization significantly diminished CCE. SRCaTs were membrane voltage-dependent. An alternate CCE measure was used to mitigate the effect of an increase in the fraction of noise in low-level fluorescence readouts during baseline measurement under hyperpolarizing conditions. C, Effect of $1 \mu \mathrm{m}$ TTX on SRCaTs. DIV 2 Lck-G-CaMP7-expressing neurons were imaged in the following experiments. The voltage-gated sodium channel blocker TTX was added to the medium at the time indicated by the arrowhead. Representative kymographs from three neurons are shown. D. Quantification of TTX effect on SRCaTs. DMI was calculated for the early phase (left: $0-5$ min after drug addition) and the late phase (5-15 min after drug addition). TTX had no effect on SRCaTs in either the early or the late phase. DMI was not significantly different from one (left; TTX early, DMI $=128 \pm 52 \%, n=14, t=2.037, p=0.0625$; right; TTX late, DMI $=192 \pm 169 \%, n=$ $14, t=2.041, p=0.0621$, one sample $t$ test against 1 , mean \pm SD). $\boldsymbol{E}$, Effect of $10 \mu \mathrm{m}$ nimodipine and $25 \mu \mathrm{M}$ FPL 64176 on SRCaTs. Drugs were added to the media at the time points indicated by arrowheads. Representative kymographs from two nimodipine-treated neurons and one FPL 64176-treated neuron are shown. SRCaTs were gradually diminished after the addition of specific L-type VGCCs blocker nimodipine. Conversely, FPL 64176, a potent activator of L-type calcium channels, enhanced SRCaT amplitude. $\boldsymbol{F}$, Effect of voltage-gated calcium channels antagonist/agonist on SRCaTs. SRCaTs were diminished by nimodipine and enhanced by FPL 64176 in both early phase (left; nimodipine, DMI $=56 \pm 40 \%, n=25, t=5.384, p<0.0001$; FPL 64176, $178 \pm 146 \%, n=16, t=2.155, p=0.0478$, one-sample $t$ test against 1 , mean \pm SD) and late phase (right; nimodipine, DMI = $48 \pm 47 \%, n=25, t=5.491, p<0.0001 ; \mathrm{FPL} 64176,158 \pm 103 \%, n=16, t=2.254, p=0.0396$, one-sample $t$ test against 1 , mean $\pm S D) .{ }^{*} p<0.05$

VGCCs are $\mathrm{Ca}_{\mathrm{v}} 1.2$ and $\mathrm{Ca}_{\mathrm{v}} 1.3$ (Striessnig et al., 2006; Vacher et al., 2008), which open minimally at resting membrane potential (Lipscombe et al., 2004).

To determine whether $\mathrm{Ca}_{\mathrm{v}} 1.2$ and $\mathrm{Ca}_{\mathrm{v}} 1.3$ were expressed during neuronal development, we performed qRT-PCR to determine mRNA expression of $\mathrm{Ca}_{\mathrm{v}} 1.2$ and $\mathrm{Ca}_{\mathrm{v}} 1.3$ compared with adult expression levels and found that the relative RNA expression levels of $\mathrm{Ca}_{\mathrm{v}} 1.2$ and $\mathrm{Ca}_{\mathrm{v}} 1.3$ in developing cortex were as high, if not higher, as in the adult. The average RNA expression level of $\mathrm{Ca}_{\mathrm{v}} 1.2$ and $\mathrm{Ca}_{\mathrm{v}} 1.3$ in developing cortex was significantly higher than that in adult cortex $(\mathrm{P} 0: 400 \pm 140 \%, \mathrm{P} 1: 370 \pm 60 \%, \mathrm{P} 2$ :

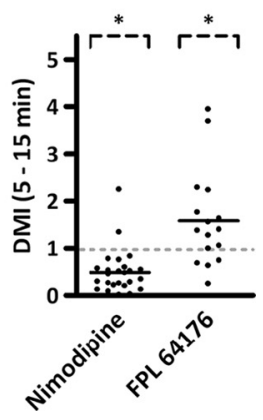

$440 \pm 130 \%, \mathrm{P} 3: 330 \pm 70 \%, \mathrm{P} 4: 390 \pm$ $70 \%$ for $\mathrm{Ca}_{\mathrm{v}} 1.2$ and $\mathrm{P} 0: 180 \pm 40 \%, \mathrm{P} 1$ : $210 \pm 20 \%, \mathrm{P} 2: 210 \pm 30 \%, \mathrm{P} 3: 190 \pm$ $10 \%, \mathrm{P} 4: 220 \pm 20 \%$ for $\mathrm{Ca}_{\mathrm{v}} 1.3$. All values are normalized to adult). Consistent with this, immunohistochemistry of mouse P1 cortical slices against $\mathrm{Ca}_{\mathrm{v}} 1.2$ and $\mathrm{Ca}_{\mathrm{v}} 1.3$ demonstrated punctate signals all over the cortex at the soma, in leading processes, and in the trailing axons (Fig. $4 A$ ). $\mathrm{Ca}_{\mathrm{v}} 1.2$ signals were more prominent than $\mathrm{Ca}_{\mathrm{v}} 1.3$ (Fig. 4A). The specificity of these antibodies were previously rigorously tested (Jing et al., 2013; Cheli et al., 2016). These signals disappeared when the antibodies were preabsorbed with the corresponding antigens. Furthermore, we verified that the immunoreactivity of $\mathrm{Ca}_{\mathrm{v}} 1.2$ antibody used here was diminished in Western blot analyses of $\mathrm{Ca}_{\mathrm{v}} 1.2$ knock-out neuronal lysates (see Fig. 6A). To confirm the presence of the predominant $\mathrm{Ca}_{\mathrm{v}} 1.2$ subunit in axons of early cortical neurons in vivo, we examined the subcellular distribution of exogenously expressed sHA-Ca 1.2 using an anti-HA tag antibody and found the HA signal of calcium channels not only in somas and dendrites but also in axons in $\mathrm{P} 1$ and $\mathrm{P} 3$ developing cortex (Fig. $4 B)$. Axonal expression of $\mathrm{Ca}_{\mathrm{v}} 1.2$ was also confirmed in P21 mouse brain using a CRSIPR/Cas9-based gene tagging technique, SLENDR, which enabled us to introduce HA tags into the C-terminal tail of endogenous $\mathrm{Ca}_{\mathrm{v}} 1.2$ (Mikuni et al., 2016) (Fig. 4C).

To determine which $\alpha 1$ subunit(s) may generate SRCaTs, we used a pharmacological knock-out system (He et al., 1997; Zhang et al., 2006) (Fig. 4D). The DHP-insensitive mutant $\mathrm{Ca}_{\mathrm{v}}$ subunits $\mathrm{Ca}_{\mathrm{v}} 1.2-\mathrm{TY}$ and $\mathrm{Ca}_{\mathrm{v}} 1.3-\mathrm{TY}$ or control DHP-sensitive $\mathrm{Ca}_{\mathrm{v}} 1.2-\mathrm{WT}$ were transfected by in utero electroporation at E15.5, along with auxiliary subunits $\mathrm{Ca}_{\mathrm{v}} \alpha 2 \delta 1, \mathrm{Ca}_{\mathrm{v}} \beta 3$, and a $\mathrm{Ca}^{2+}$ reporter Lck-G-CaMP7. SRCaTs were then imaged in the presence of $10 \mu \mathrm{M}$ nimodipine to discriminate endogenous SRCaTs through WT DHP-sensitive $\mathrm{Ca}_{\mathrm{v}}$ 1.2/1.3 from those driven by exogenous DHP-resistant $\mathrm{Ca}_{\mathrm{v}} 1.2 / 1.3$ subunits. We found that nimodipine, which significantly diminished SRCaTs when $\mathrm{Ca}_{\mathrm{v}} 1.2 / 1.3$ was WT and DHP-sensitive, was unable to block SRCaTs when a DHP-resistant $\mathrm{Ca}_{\mathrm{v}} 1.2$ or $\mathrm{Ca}_{\mathrm{v}} 1.3$ was expressed (Fig. $4 E$ ). These results demonstrated that expression of $\mathrm{Ca}_{\mathrm{v}} 1.2$ or $\mathrm{Ca}_{\mathrm{v}} 1.3$ L-type VGCCs was sufficient to trigger SRCaTs in immature neurons, although the extent of Cav1.2 or Cav1.3 expression sufficient for triggering SRCaTs remains to be determined. Next, we investigated whether SRCaTs-like calcium elevations were modulated by the kinetics and amplitude of $\mathrm{Ca}_{\mathrm{v}}$ currents. Intriguingly, SRCaTs mediated by gain-of-function $\mathrm{Ca}_{\mathrm{v}} 1.2-\mathrm{G} 406 \mathrm{R}$ channels showed 


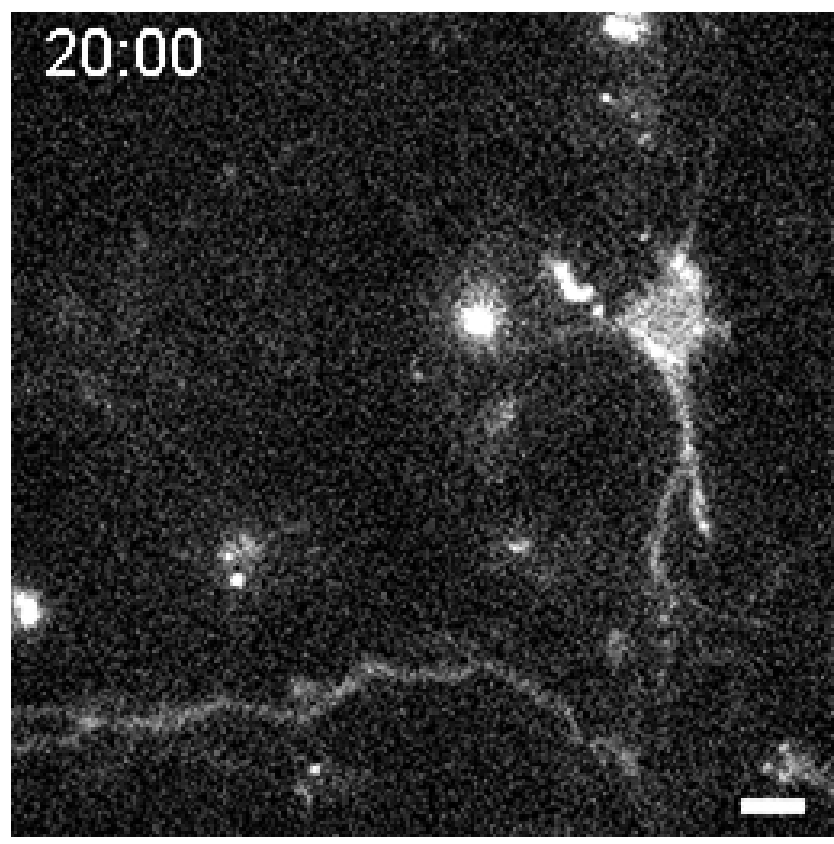

Movie 4. Representative response to $10 \mu \mathrm{M}$ nimodipine. DIV 2 LckG-CaMP7-expressing neurons were treated by nimodipine. The drug was added 10 minutes after the start of image acquisition at $10 \mu \mathrm{M}$. Images were acquired every $1 \mathrm{~s}$ at 25 frames/s. Scale bar, $5 \mu \mathrm{m}$.

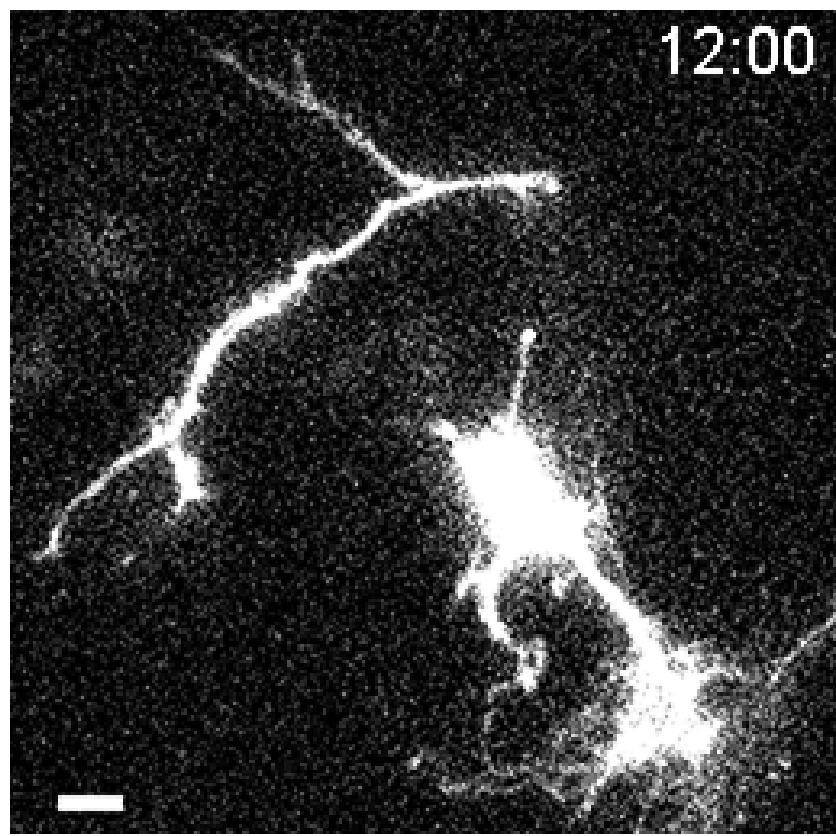

Movie 5. Representative response to $25 \mu \mathrm{M}$ FPL 64176. DIV 2 Lck G-CaMP7-expressing neurons were treated by FPL 64176. The drug was added 10 minutes after the start of image acquisition at $25 \mu \mathrm{M}$. Images were acquired every $1 \mathrm{~s}$ at 25 frames/s. Scale bar, $5 \mu \mathrm{m}$.

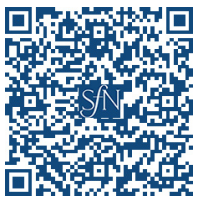

significantly larger spatial spreads and amplitude than those of $\mathrm{Ca}_{\mathrm{v}} 1.2$-WT (Fig. $5 A, C$ ). However, surprisingly, the duration of calcium elevation was shorter (Fig. $5 B$ ). Reducing $\mathrm{Ca}^{2+}$ permeation through additional 4EQ mutations $\left(\mathrm{Ca}_{v} 1.2-\mathrm{G} 406 \mathrm{R}-\right.$
$4 \mathrm{EQ}$ ) reversed the effects on spatial spread and duration near to WT levels, although a significant SRCaT amplitude was still recorded, perhaps due to a conformational effect (Fig. $5 A-C$ ).

Neurodevelopmental phenotypical signatures of $\mathrm{Ca}_{\mathrm{v}} 1.2$ in perinatal corticogenesis

To demonstrate a perinatal neurodevelopmental relevance of $\mathrm{Ca}_{\mathrm{v}} 1.2 / 1.3$ subunits, we transfected Cre recombinase into embryonic cortical neurons of floxed $\mathrm{Ca}_{\mathrm{v}} 1.2$ mice by in utero electroporation (Seisenberger et al., 2000). pCAG-EGFP-KRasCT (control) or pCAG-Cre-P2A-mCherry-KRasCT (knock-out) plasmids were introduced into E12.5 $\mathrm{Ca}_{\mathrm{v}} 1.2$ conditional knock-out (cKO) homozygote embryos. Cortical neurons were prepared from E18 embryos and neurons marked with either EGFP (Cre-nonexpressing) or mCherry (Cre-expressing) were mixed with nontransfected neurons and plated onto the same coverslips to minimize biases due to neuronal culture density. As shown in Figure $6 B$, both $\mathrm{Ca}_{\mathrm{v}} 1.2$ pharmacology and genotype significantly affected the neurite length. The knock-out of endogenous $\mathrm{Ca}_{\mathrm{v}} 1.2$ reduced total neurite length in the drug-free condition, consistent with a promoting effect of $\mathrm{Ca}^{2+}$ signaling on immature neurite elongation. Although nimodipine had little additional effect on $\mathrm{Ca}_{\mathrm{v}} 1.2$ knocked-out neurons, FPL 64176, an activator of L-type calcium channels, still marginally affected the neurite length in the knock-out condition, presumably through an effect on $\mathrm{Ca}_{\mathrm{v}} 1.3$. Cre expression per se did not cause any effect (Fig. 6C). Together, these results demonstrated the physiological importance of $\mathrm{Ca}_{\mathrm{v}} 1.2$ for neurite elongation in perinatal brains.

Having firmly established that $\mathrm{Ca}_{\mathrm{v}} 1.2$ was expressed in young cortical neurons and promoted immature neurite growth, we next sought for other functions of $\mathrm{Ca}_{\mathrm{v}} 1$-type VGCCs during development. In particular, we were interested in radial migration because it is a key step for cortical formation. To address this, we introduced WT and mutant $\mathrm{Ca}_{\mathrm{v}} 1.2$ subunits, along with auxiliary subunits and a fluorescent marker in future layer $2 / 3$ excitatory cortical neurons through in utero electroporation at E15.5. We compared the effect of control (transfection marker, auxiliary subunit only), $\mathrm{Ca}_{\mathrm{v}} 1.2-\mathrm{WT}$ - and gain-of-function $\mathrm{Ca}_{\mathrm{v}} 1.2-\mathrm{G} 406 \mathrm{R}$ expression on radial migration of layer $2 / 3$ neurons (Fig. $7 A$ ). Comparable expression of the exogenous channels was confirmed by immunohistochemistry against HA and ectopic neurons were identified and counted as cells located below the destined layer $2 / 3$ at P16.

Forced expression of WT $\mathrm{Ca}_{\mathrm{v}} 1.2$ slightly but significantly impaired radial migration, whereas introduction of the $\mathrm{Ca}_{\mathrm{v}} 1.2-\mathrm{G} 406 \mathrm{R}$ mutant caused a much more severe phenotype (Fig. $7 B, C$ ). Reducing $\mathrm{Ca}^{2+}$ permeation in the forcefully expressed $\mathrm{Ca}_{\mathrm{v}} 1.2-\mathrm{G} 406 \mathrm{R}$ subunit with $4 \mathrm{EQ}$ mutations abolished the migration deficit (Fig. $7 C$ ), suggesting that aberrant calcium overload via $\mathrm{Ca}_{\mathrm{v}} 1.2$ indeed disrupted the proper migration process in a calcium-dosedependent manner.

Next, we tried to determine the developmentally sensitive period of this $\mathrm{Ca}_{\mathrm{v}} 1.2$-mediated calcium overload on the radial migration. In contrast to forced expression using a constitutive CAG promoter, we induced the expression of $\mathrm{Ca}_{\mathrm{v}} 1.2-\mathrm{G} 406 \mathrm{R}$ postnatally using the Tet-ON TRE/rtTA system (Fig. 7D). Postnatal expression of $\mathrm{Ca}_{\mathrm{v}} 1.2-\mathrm{G} 406 \mathrm{R}$ showed little perturbation on radial migration compared with $\mathrm{Ca}^{2+}$-nonpermeating $\mathrm{Ca}_{\mathrm{v}} 1.2-\mathrm{G} 406 \mathrm{R}$ 4EQ controls. This indicated that prenatal, but not postnatal, maladaptive calcium elevation was causing a radial migration defect (Fig. 7D). 
A
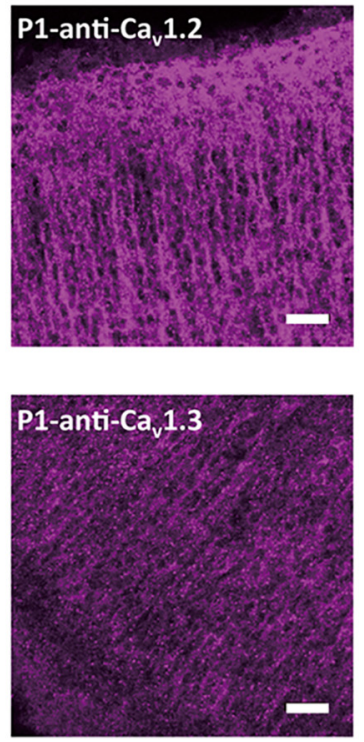

B
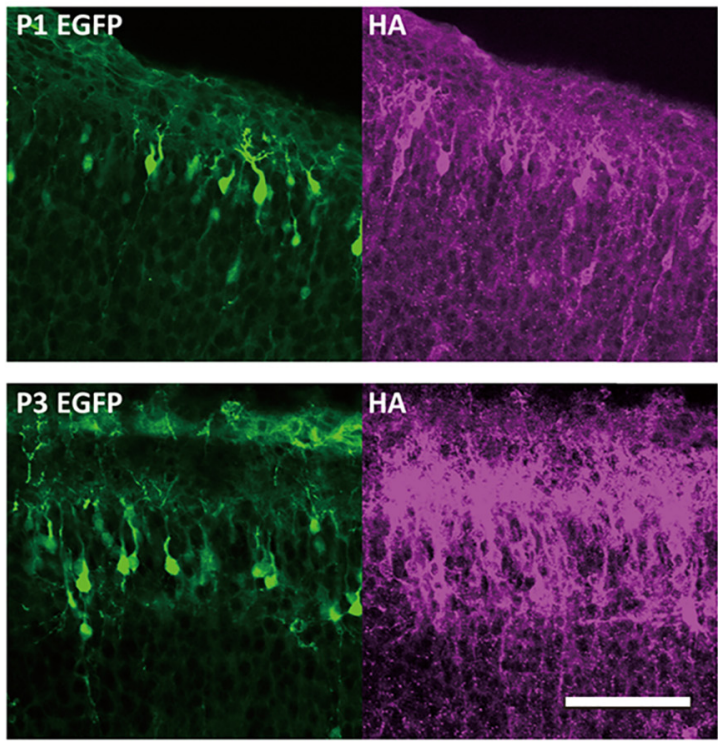

C

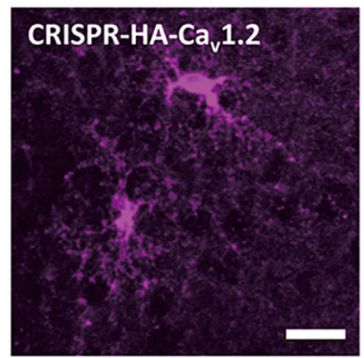

D

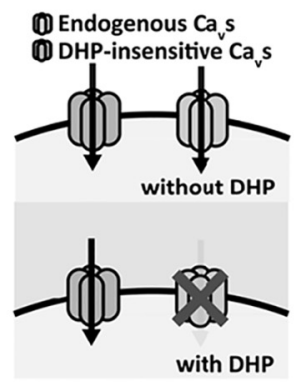

$\mathbf{E}$

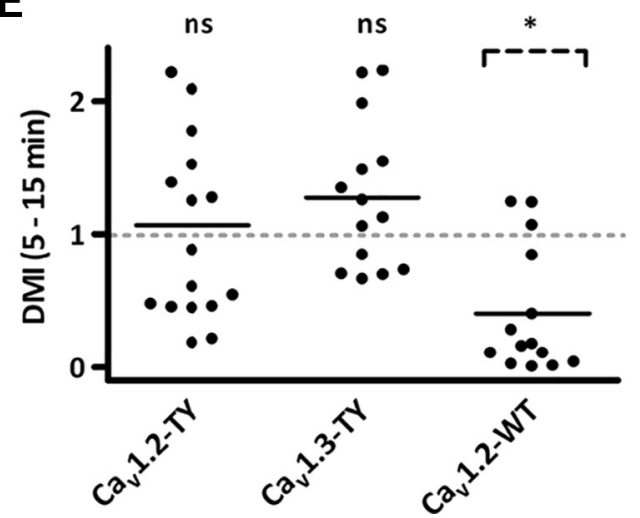

Figure 4. Exogenously expressed DHP-insensitive L-type VGCCs $\alpha$ subunits replicated SRCaTs in vitro. A, Immunohistochemistry of L-type calcium channels in P1 mouse cortex. Anti-Ca 1.2 (left) and anti-Ca 1.3 (right) P1 mouse cortical sections were immunostained against $\mathrm{Ca}_{v} 1.2$ and $\mathrm{Ca}_{v} 1.3$. Immunohistochemistry showed that both subtypes were expressed in developing mouse cortex. Fewer punctate signals were observed in $\mathrm{Ca}_{\mathrm{v}} 1.3$ staining condition. Scale bar $20 \mu \mathrm{m}$. B, Subcellular localization of exogenously expressed Ca 1.2 . pCAG-SHA-Ca $1.2-T Y-G 406 R-4 E Q$, pCAG-EGFP and auxiliary subunits were introduced into future cortical layer 2/3 neurons by in utero electroporation at E15.5. Brain sections were immunostained against EGFP (green) and HA (magenta). Although immunofluorescence was mainly localized in somatic and dendritic region, there were weaker punctate signals on axons both in P1 (top) and P3 (bottom) slices. The subcellular localization of calcium channels in situ was in accord with the finding that SRCaTs were observed in both axons and dendrites. Scale bar, $80 \mu \mathrm{m}$. C, Subcellular localization of endogenous $\mathrm{Ca}_{\mathrm{v}} 1.2$ labeled by SLENDR technique. pCAG-hSpCas9-Ca 1 1.2, pCAG-EGFP-KRasCT and cognate ss0DNs mixture was transfected by in utero electroporation at E12.5. P21 cortical sections were immunostained against HA. Due to relatively low expression level, we could not find any HA-positive neurons in the sections from earlier developing stages (P1 to P7). HA-signals (magenta) were detected in entire neurites. The distribution of endogenous $\mathrm{Ca}_{\mathrm{v}} 1.2$ was consistent with the spatial pattern of SRCaTs despite the difference in developing stages. Maximum stack of z-projection. Scale bar, $20 \mu \mathrm{m}$. D. Schematics of pharmacological knock-out. DHP-insensitive L-type VGCCS were expressed in neurons (top) then treated with DHPs (bottom). Selective inhibition of endogenous channels enabled us to isolate the function of exogenously expressed channels. $E$, Effects of exogenously expressed L-type calcium channels on SRCaTs. SRCaTs were not suppressed in DHP-insensitive-channel-expressing neurons (sHA-Ca 1 1.2-T1039Y, HA-Ca $1.3-\mathrm{T} 1033 \mathrm{Y}$ ). However, they were significantly diminished in DHP-sensitive channel (sHA-Ca 1 1.2-WT)-expressing neurons ( $\mathrm{Ca}_{\mathrm{v}} 1.2-\mathrm{TY}, \mathrm{DMI}=107 \pm 73 \%, n=17, t=0.384, p=0.7060$; $\mathrm{Ca}_{\mathrm{v}} 1.3-\mathrm{TY}$, $\mathrm{DMI}=127 \pm 55 \%, n=14, t=1.848, p=0.0875 ; \mathrm{Ca}_{\mathrm{v}} 1.2-\mathrm{WT}$, DMI $=40 \pm 47 \%, n=14, t=4.698, p=0.0004$; one-sample $t$ test against 1, mean \pm SD). It was shown that both $\mathrm{Ca}_{\mathrm{v}} 1.2$ and $\mathrm{Ca}_{\mathrm{v}} 1.3$ were capable of mimicking SRCaTs in developing neurons near resting membrane voltage. ${ }^{*} p<0.05$; ns, not significant.

We further hypothesized that, if we corrected the prenatally induced $\mathrm{Ca}_{\mathrm{v}} 1.2$-mediated $\mathrm{Ca}^{2+}$ overload after birth by postnatally shutting down $\mathrm{Ca}_{\mathrm{v}} 1.2-\mathrm{G} 406 \mathrm{R}$ expression, we might rescue the migration deficit. To test this directly, we initially forcefully expressed $\mathrm{Ca}_{\mathrm{v}} 1.2-\mathrm{G} 406 \mathrm{R}$ before birth using a Tet-OFF TRE/tTA system and then eliminated postnatal $\mathrm{Ca}_{\mathrm{v}} 1.2-\mathrm{G} 406 \mathrm{R}$ expression by postnatal doxycycline treatment. Consistent with CAG promoter-mediated overexpression (Fig. $7 B, C$ ), prenatal and postnatal overexpression of $\mathrm{Ca}_{\mathrm{v}} 1.2-\mathrm{G} 406 \mathrm{R}$ in the absence of doxycycline treatment led to significant migration deficit (Fig. $7 E$, left). However, turning off $\mathrm{Ca}_{\mathrm{v}} 1.2-\mathrm{G} 406 \mathrm{R}$ expression post- natally using doxycycline resulted in a loss of phenotype such that we were unable to detect any difference between the $\mathrm{Ca}_{\mathrm{v}} 1.2-\mathrm{G} 406 \mathrm{R}-$ and $\mathrm{Ca}_{\mathrm{v}} 1.2-\mathrm{G} 406 \mathrm{R}-4 \mathrm{EQ}$-expressing conditions (Fig. $7 E$, right).

Together, these results provide compelling evidence that impaired radial migration was caused by aberrant calcium influx during a prenatal migration period via $\mathrm{Ca}_{\mathrm{v}} 1.2$-mediated overload. When such calcium maladaptation was removed postnatally, migratory defects became undetectable, suggesting that stalled ectopic neurons could catch up with normally migrated neurons or were extinguished because they could not survive in 
A

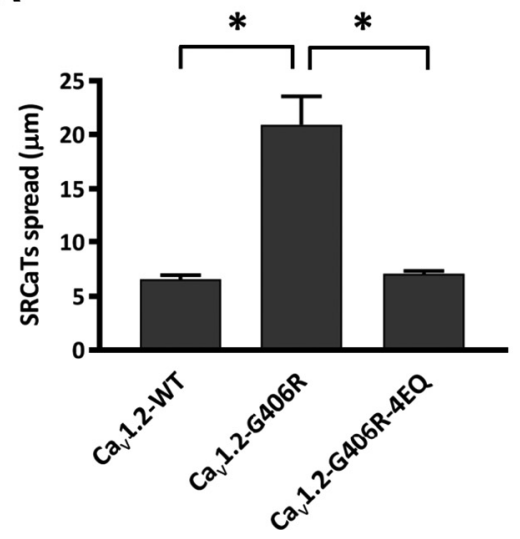

B

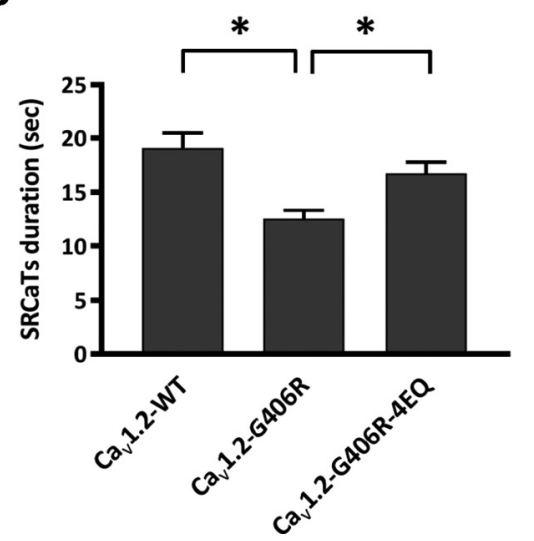

C

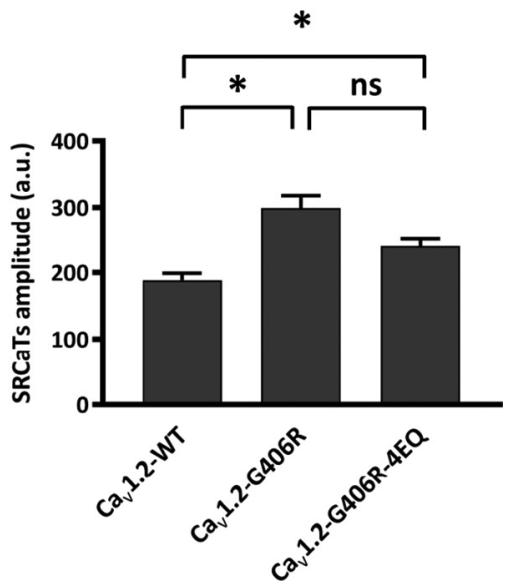

Figure 5. Bidirectional modulation of SRCaTs by $\mathrm{Ca}_{\mathrm{v}} 1.2$ mutations that affect channel inactivation and permeation. All calcium channels shown in this figure harbor nimodipine-insensitive mutations. A, Spatial spread of SRCaTs (Ca $1.2-$ WT, $6.56 \pm 0.36 \mu \mathrm{m}, n=100 ; \mathrm{Ca}_{\mathrm{v}}$ 1.2-G406R, $20.93 \pm 2.59 \mu \mathrm{m}, n=134 ; \mathrm{Ca}_{\mathrm{v}}$ 1.2-G406R-4EQ, 7.04 $\pm 0.30 \mu \mathrm{m}, n=154 ;$ Kruskal-Wallis statistic $=38.53, p<0.0001$, Kruskal-Wallis test, mean \pm SEM; Ca $1.2-$ WT vs Ca $1.2-G 406 R$, mean rank difference $=-82.46, p<0.0001 ; C_{v} 1.2-W T$ vs Ca 1 1.2-G406R-4EQ, mean rank difference $=-15.29, p>0.05 ; \mathrm{Ca}_{v} 1.2-\mathrm{G} 406 \mathrm{R}$ vs $\mathrm{C}_{\mathrm{v}} 1.2-\mathrm{G} 406 \mathrm{R}-4 \mathrm{EQ}$, mean rank difference $=67.17, p<0.0001$; post hoc Dunn's multiple-comparisons test). $\boldsymbol{B}$, Duration of SRCaTs (Ca 1.2 -WT, $19.07 \pm 1.40 \mathrm{~s}, n=100 ; \mathrm{Ca}_{\mathrm{v}} 1.2-\mathrm{G} 406 \mathrm{R}, 12.54 \pm 0.79 \mathrm{~s}, n=134 ; \mathrm{Ca}_{\mathrm{v}} 1.2-\mathrm{G} 406 \mathrm{R}-4 \mathrm{EQ}, 16.74 \pm 1.04 \mathrm{~s}, n=154 ;$ Kruskal-Wallis statistic $=19.94, p<0.0001$, Kruskal-Wallis test, mean \pm SEM;

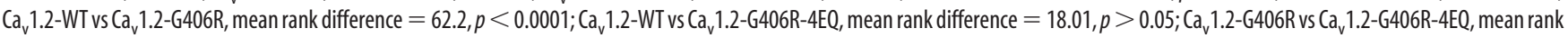
difference $=-44.19, p<0.01$; post hoc Dunn's multiple-comparisons test). C, Amplitude of SRCaTs (Ca 1 1.2-WT, $188.6 \pm 10.4, n=100 ; C_{v}$ 1.2-G406R, 298.9 $\pm 18.6, n=134 ; C_{v}$ 1.2-G406R4EQ, 239.9 $\pm 11.0, n=154 ;$ Kruskal-Wallis statistic $=15.33, p=0.0005$, Kruskal-Wallis test, mean \pm SEM; $C_{\mathrm{v}} 1.2-$ WT vs Ca $1.2-$ G406R, mean rank difference $=-56.88, p<0.001 ; C_{v} 1.2-$ WT vs $\mathrm{C}_{v} 1.2-\mathrm{G} 406 \mathrm{R}-4 \mathrm{EQ}$, mean rank difference $=-41.61, p<0.05$; $\mathrm{C}_{v}$ 1.2-G406R vs $\mathrm{Ca}_{v} 1$.2-G406R-4EQ, mean rank difference $=15.28, p>0.05$; post hoc Dunn's multiple-comparisons test). Spatiotemporal features of SRCaTs were accentuated by a gain-of-function G406R mutation and reversed by $\mathrm{Ca}^{2+}$-permeation-reducing 4 EQ mutations, compared with WT $\mathrm{Ca}_{\mathrm{v}} 1.2$, demonstrating a causal link between $\mathrm{Ca}_{\mathrm{v}} 1.2$ and SRCaTs. However, in one parameter (SRCaT amplitude), we found a lack of statistical significance between $\mathrm{Ca}_{\mathrm{v}} 1.2-\mathrm{G} 406 \mathrm{R}$ with or without 4EQ mutations, indicating a $\mathrm{Ca}^{2+}$-independent, conformation-dependent effect of $\mathrm{Ca}_{\mathrm{v}} 1.2$ on SRCaT as well. ${ }^{*} p<0.05$; ns, not significant.

A

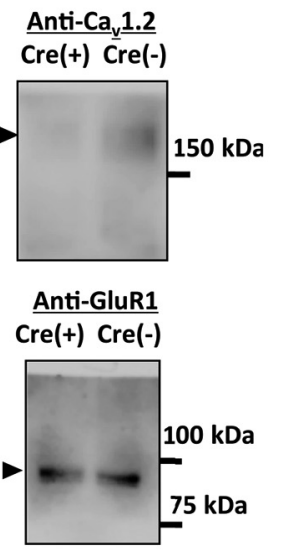

B

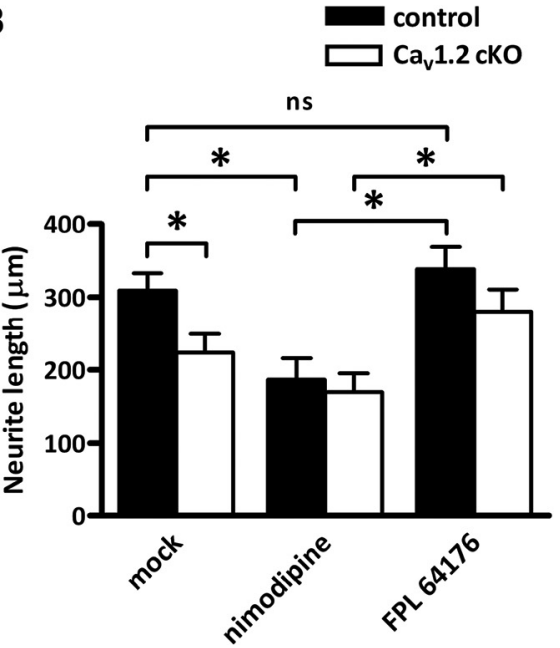

C

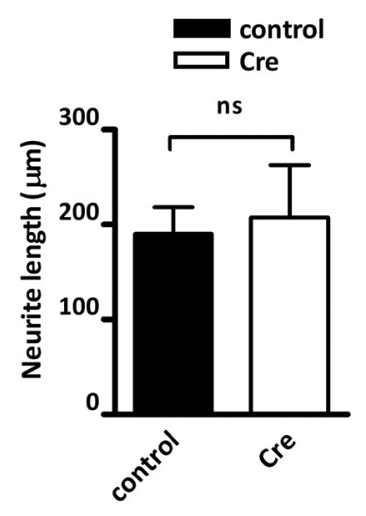

Figure 6. Alteration of neurite length by modulation of endogenous L-type calcium channels. A, Representative Western blot analysis of proteins extracted from Cacna 1 c conditional knock-out mice cortical neurons. Anti-Ca 1.2 (CACNA1C) intensity was attenuated (to 25\%) in Cre-expressing condition compared with noninfected counterpart. Anti-Ca 1.2 (top) and anti-GluR1 (bottom) immunoreactivities were compared in the upper (top) or lower (bottom) halves of the same blot. $\boldsymbol{B}$, Effects of drugs on neurite length in $\mathrm{Ca}_{\mathrm{v}} 1.2$ conditional knock-out background. Transfection marker pCAG-EGFP-KRasCT or pCAG-Cre-P2A-mCherry-KRasCT was transfected by in utero electroporation into Ca 1.2 conditional knock-out mice embryos at E12.5. Dissociated EGFP- or Cre-P2A$\mathrm{mCherry-KRasCT-expressing} \mathrm{neurons} \mathrm{were} \mathrm{mixed} \mathrm{and} \mathrm{plated} \mathrm{onto} \mathrm{coverslips} \mathrm{and} \mathrm{drugs} \mathrm{were} \mathrm{added} \mathrm{after} 6 \mathrm{~h}$. The neurons were fixed $48 \mathrm{~h}$ after plating and immunoenhanced with antibodies. The length of neurites was quantified (WT-mock, $309 \pm 24 \mu \mathrm{m}, n=14$; WT-nimodipine, $187 \pm 29 \mu \mathrm{m}, n=12$; WT-FPL 64176, $338 \pm 30 \mu \mathrm{m}, n=13$; K0-mock, $224 \pm 25 \mu \mathrm{m}, n=15$; K0-nimodipine, $170 \pm 25 \mu \mathrm{m}, n=10$; KO-FPL 64176, $281 \pm 30 \mu \mathrm{m}, n=10$; drug effect, $F_{(2.69)}=11.60, p<0.0001$; genotype effect, $F_{(1,69)}=5.51, p=0.0218$, two-way ANOVA, mean \pm SEM) Comparison between genotype was performed in mock condition (WT-mock vs KO-mock; Mann-Whitney U $=53, p=0.0229$, Mann-Whitney test). Importantly, knock-out of Ca 1.2 resulted in shorter neurites. In EGFP transfected neurons, the total neurite length was significantly shortened by nimodipine whereas the proelongation effect of FPL 64176 was not clear (WT, Kruskal-Wallis stastic $=11.57, p=0.0031$, Kruskal-Wallis test; mock vs FPL 64176, mean rank difference $=-0.846, p>0.05$; mock vs nimodipine, mean rank difference $=13.09, p<0.05 ; \mathrm{FPL} 64176$ vs nimodipine, mean rank difference $=13.94, p<0.01$; post hoc Dunn's multiple-comparisons test). Although $\mathrm{Ca}_{\mathrm{v}} 1.2$ was knocked-out, the effect of L-type calcium channel modulators was marginally significant (KO, Kruskal-Wallis statistic $=5.991, p=0.0500$, Kruskal-Wallis test; mock vs FPL 64176, mean rank difference $=-6.067, p>0.05$; mock vs nimodipine, mean rank difference $=5.133, p>0.05 ; \mathrm{FPL} 64176$ vs nimodipine, mean rank difference $=11.2, p<0.05 ;$ post hoc Dunn's multiple-comparisons test). $C$, Effects of (re expression on neurite length in WT background. pCAG-EGFP-KRasCT or pCAG-Cre-P2A-mCherry-KRasCT were transfected into WT C57/B6J cortical neurons by in utero electroporation at E12.5. The length of neurites was quantified as in $A$, (EGFP, $305 \pm 30 \mu \mathrm{m}, n=13$; (re-P2A-mCherry, $332 \pm 56 \mu \mathrm{m}, n=6 ; t=0.309, p=0.7614$, unpaired $t$ test, mean \pm SEM). ${ }^{*} p<0.05$; ns, not significant. 
A

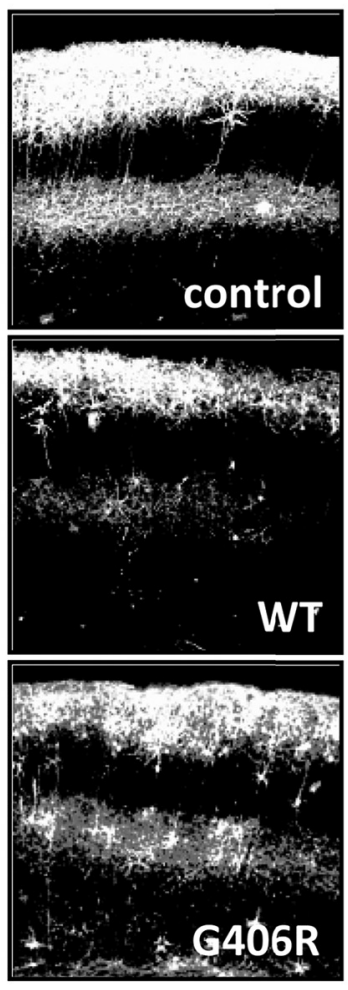

D

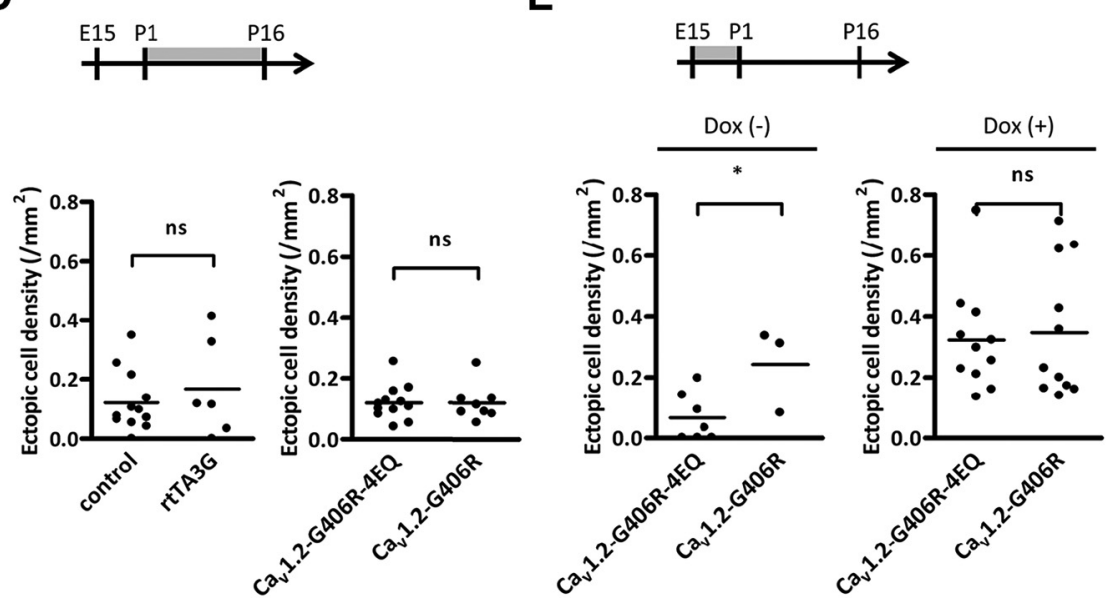

Figure 7. Impaired neuronal migration is caused by perinatal $\mathrm{Ca}^{2+}$ overload through misregulated L-type VGCCs and can be postnatally corrected. $\boldsymbol{A}$, Representative images of P16 brain slices. pCAG-SHA-Ca 1 .2-WT or its mutant, pCAG-EGFP, and auxiliary subunits were transfected by in utero electroporation at E15.5. Ca 1.2 vector was substituted with pCAG-pro-C1 empty vector in control condition. Calcium channels were constitutively expressed from E15.5 to P16. Control (top), Ca $1.2-W T$ (middle), and $\mathrm{Ca}_{v} 1.2-\mathrm{G} 406 \mathrm{R}$ (bottom) are shown. Overexpression of calcium channel impaired proper radial migration. All sections were immunoenhanced with anti-GFP antibody. B, Quantification of ectopic cell density in CAG-Ca 1 .2-expressing conditions. Control versus $C_{v} 1.2-W T$ (left), control versus C $a_{v} 1.2-G 406 R$ (middle), and Ca $1.2-W T$ versus $C_{v} 1.2-G 406 R$ (right) are shown. C $a_{v} 1.2$ or its mutant was transfected by in utero electroporation at E15.5. Channels were constitutively expressed from E15.5 to P16. To reduce possible variability introduced by transfection efficiency, birth timing difference, and subtle immunohistochemistry conditions, we compared the pups from the same animal side by side. Exogenous overexpression of $C a_{v} 1.2$ significantly increased ectopic cell density (control, $0.45 \pm 0.13 / \mathrm{mm}^{2}, n=12 ; \mathrm{Ca}_{\mathrm{v}} 1.2-\mathrm{WT}, 2.09 \pm 0.46 / \mathrm{mm}^{2}, n=9 ; t=3.851, p=0.0011$, unpaired two-tailed $t$ test; control, $0.29 \pm 0.10 / \mathrm{mm}^{2}, n=9 ; \mathrm{Ca}_{\mathrm{v}} 1.2-\mathrm{G} 406 \mathrm{R}, 3.55 \pm 0.84 / \mathrm{mm}^{2}, n=9 ; t=3.862, p=0.0014$, unpaired two-tailed $t$ test; $\mathrm{Ca}_{\mathrm{v}} 1.2-\mathrm{WT}, 0.93 \pm 0.87 / \mathrm{mm}^{2}, n=10 ; \mathrm{Ca}_{\mathrm{v}} 1.2-\mathrm{G} 406 \mathrm{R}, 8.38 \pm 3.30 / \mathrm{mm}^{2}, n=7 ; t=6.892, p<0.0001$, unpaired two-tailed $t$ test, mean \pm SD). Ectopic cell density was highest in G406R, Timothy mutant expressing condition. Expression of WT $\mathrm{Ca}_{\mathrm{v}} 1.2$ gave less ectopic cells, which implied the amount of calcium influx correlated the severity of the phenotype. Light gray bars represent the period when the promoter is active in the following figures. $\mathbf{C}$, Comparison among control, CAG-Ca $1.2-\mathrm{G} 406 \mathrm{R}$ and CAG-Ca 1 1.2-G406-4EQ expressed conditions. Control versus Ca $1.2-G 406 R$ (left) and Ca $1.2-G 406 R-4 E Q$ versus Ca $1.2-G 406 R$ (right) are shown. The channels shown above were constitutively expressed from E15.5 to P16. Calcium impermeable 4EQ pore the cortical circuit. We thus conclude that a prenatal neurodevelopmental effect of $\mathrm{Ca}_{\mathrm{v}} 1.2$-mediated overload could be rescued postnatally by correcting the $\mathrm{Ca}_{\mathrm{v}} 1.2$ dysfunction.

\section{Discussion}

Prime role for L-type calcium channels in determining SRCaTs in immature neurons

We visualized the dynamic spatiotemporal nature of SRCaTs in immature cortical neurons by combining in utero electroporation with membrane-tethered G-CaMP7. Pharmacological experiments revealed that the primary $\mathrm{Ca}^{2+}$ source of SRCaTs was L-type calcium channels. This was a surprising result because L-type calcium channels rarely open near resting potential (Lipscombe et al., 2004). Although TTX had no effect, $\mathrm{K}_{\mathrm{ir}} 2.1$ expression blocked SRCaTs, suggesting that a depolarizing shift of resting membrane potentials,

$\leftarrow$

mutant could not impair migration (control, $0.12 \pm 0.03$ $/ \mathrm{mm}^{2}, n=12 ; \mathrm{Ca}_{\mathrm{v}} 1.2-\mathrm{G} 406 \mathrm{R}, 1.09 \pm 0.14 / \mathrm{mm}^{2}, n=9 ; t=$ 7.78, $p<0.0001$, unpaired two-tailed $t$ test; $\mathrm{Ca}_{\mathrm{v}} 1.2-\mathrm{G} 406 \mathrm{R}$ $4 \mathrm{EQ}, 0.12 \pm 0.02 / \mathrm{mm}^{2}, n=12 ; \mathrm{Ca}_{\mathrm{v}} 1.2-\mathrm{G} 406 \mathrm{R}, 0.41 \pm 0.10$ $/ \mathrm{mm}^{2}, n=7 ; t=3.787, p=0.0015$, unpaired two-tailed $t$ test, mean \pm SD). Although it is known that calcium channels can transmit signals regardless of calcium influx into cells, according to this figure, migration defects were actually caused by calcium influx through L-type calcium channels. D, Postnatally induced conditions (Tet-on with TRE-Ca 1.2 ). The expression of calcium channels was not constitutive but induced by postnatal doxycycline administration to mother mice. pCAG$s H A-C a_{v} 1.2$ or its mutants were substituted with the mixture of pCAG-rtTA3G (Tet-on) and TRE-sHA-Ca 1.2 or its variants. Channels were inducibly expressed from P1 to P16. TRE$C a_{v} 1.2-G 406 R$ without versus with rtTA3G (left) and TRE$\mathrm{Ca}_{v} 1.2-\mathrm{G} 406 \mathrm{R}$ versus 4EQ (right) are shown. Postnatally expressed calcium channels could not impair radial migration (without rtTA, $0.12 \pm 0.03 / \mathrm{mm}^{2}, n=12$; with rtTA, $0.17 \pm$ $0.07 / \mathrm{mm}^{2}, n=9 ; t=0.730, p=0.4762$, unpaired twotailed $t$ test; TRE-Ca $1.2-$ G406R-4EQ, $0.12 \pm 0.02 / \mathrm{mm}^{2}, n=$ $12 ; \mathrm{Ca}_{v} 1.2-\mathrm{G} 406 \mathrm{R}, 0.12 \pm 0.02 / \mathrm{mm}^{2}, n=8 ; t=0.026, p=$ 0.9797 , unpaired two-tailed $t$ test, mean \pm SD). $\boldsymbol{E}$, Postnatally suppressed conditions (Tet-off with TRE-Ca 1.2 ). The pCAGrtTA3G plasmid used in Figure 7D was substituted with pCAGtTA3G (Tet-off). Channels were expressed from E15.5 to P1-P3 (immunohistochemistry showed that most exogenous channels were degraded $2 \mathrm{~d}$ after doxycycline administration, unpublished data). Without doxycycline (left) and TRE-Ca $1.2-$ G406R versus 4EQ (right). Without doxycycline, L-type calcium channels are continuously expressed. The observed phenotype was similar to that of constitutively expressed conditions. (TRE-Ca 1 1.2-G406R-4EQ, $0.07 \pm 0.08 / \mathrm{mm}^{2}, n=7$; TRECav1.2-G406R, $0.24 \pm 0.14 / \mathrm{mm}^{2}, n=3 ; t=2.606, p=$ 0.0313 , unpaired two-tailed $t$ test, mean \pm SD). TRE- $C a_{v} 1.2-$ G406R versus 4EQ (right) is shown (TRE-Ca $1.2-\mathrm{G} 406 \mathrm{R}-4 \mathrm{EQ}$, $0.32 \pm 0.05 / \mathrm{mm}^{2}, n=11 ; \mathrm{Ca}_{\mathrm{v}} 1.2-\mathrm{G} 406 \mathrm{R}, 0.35 \pm 0.07$ $/ \mathrm{mm}^{2}, n=11 ; t=0.295, p=0.7712$, unpaired two-tailed $t$ test, mean \pm SD). Postnatal suppression of $\mathrm{Ca}_{\mathrm{v}} 1.2$ expression was enough for the stalled neurons to catch up with normally migrated neurons. ${ }^{*} p<0.05$; ns, not significant. 
driven either spontaneously or via effects of extracellular signals, might account for the apparently paradoxical opening of L-type calcium channels. We failed to detect any voltage fluctuation through voltage imaging using the genetically encoded voltage indicator ArcLight (Jin et al., 2012) or its faster variants. However, we cannot exclude that spatially and temporally restricted peaks of local voltage fluctuations may have escaped our measurements because they are either too subtle or too localized to be detected using currently available voltage-sensing probes. Differential splicing (Lipscombe et al., 2002; Liu et al., 2003) or distinct subunit composition of $\mathrm{Ca}_{\mathrm{v}} 1.2$ and $\mathrm{Ca}_{\mathrm{v}} 1.3$ (Hetzenauer et al., 2006; Striessnig et al., 2006) may also alter the biophysical properties of L-type channels in developing cortical neurons in a manner distinct from those found in adult channels. Indeed, some L-type calcium channels may open at relatively negative potential (Xu and Lipscombe, 2001) and some neuronal L-type channels have different opening properties from well studied cardiac channels (Helton et al., 2005).

We confirmed that both $\mathrm{Ca}_{\mathrm{v}} 1.2$ and $\mathrm{Ca}_{\mathrm{v}} 1.3$ were expressed in neurons of developing cortex; however, it remains to be seen whether SRCaT effect of both channels can be differently decoded locally, perhaps by distinct downstream effector molecules, or if widespread $\mathrm{Ca}^{2+}$ signals downstream of either channel constitute the crux of SRCaTs. There are precedents for locationspecific $\mathrm{Ca}^{2+}$ signaling in axons and dendrites via differential usage of CaMKI isoforms (Takemoto-Kimura et al., 2007; AgetaIshihara et al., 2009). Experimentally, however, we were unable to physically disambiguate SRCaTs derived from either $\mathrm{Ca}_{\mathrm{v}} 1.2$ or $\mathrm{Ca}_{\mathrm{v}} 1.3$ channels in living neurons. Furthermore, either channel, when expressed exogenously, was sufficient to drive SRCaTs and both seemed to contribute to the neurite growth effects downstream of SRCaTs. We speculate that, in principle, information might be encoded in SRCaTs either in the amplitude and/or frequency domains, both of which were severely affected by $\mathrm{Ca}_{\mathrm{v}} 1.2$ mutations. These parameters then may dictate which downstream molecules were ultimately responsible for coupling developmental $\mathrm{Ca}^{2+}$ signals into long-term changes in gene expression or morphology (Bito et al., 1996; Deisseroth et al., 1996; Dolmetsch et al., 1997; Wheeler et al., 2012; Fujii et al., 2013).

Because we used cultured neurons, an ultimate criticism is that the membrane integrity may be damaged during the dissociation process. Therefore, results should be interpreted with cautions, especially for neurons immediately after plating. The bidirectional and reversible nature of the effects of $\mathrm{Ca}_{\mathrm{v}} 1.2$ mutations, however, largely mitigate this concern. In addition, preliminary observations suggest the possibility that SRCaT-like calcium elevations may also operate in mouse cortical slices (unpublished observation). Therefore, it will be of great interest to test, in future work, the in vivo functions of SRCaT-like activities. Furthermore, the overall complexity and physiological landscape of L-type calcium channel activity that may critically contribute to neurodevelopmental processes will need to be fully elucidated.

\section{Neurodevelopmental consequences of prenatal $\mathrm{Ca}_{\mathrm{v}} \mathrm{1} .2$ malfunction may be corrected postnatally}

In this work, the identification of $\mathrm{Ca}_{\mathrm{v}} 1.2$ as one of the likely source of SRCaTs' $\mathrm{Ca}^{2+}$ entry prompted us to investigate the neurodevelopmental consequences of either upregulating or downregulating its $\mathrm{Ca}^{2+}$ influx using genetic means. Surprisingly, our findings showed that $\mathrm{Ca}_{\mathrm{v}} 1.2$ activity showed a positive effect on the elongation of neurites. Furthermore, we found that maladaptive $\mathrm{Ca}^{2+}$ influx, through mutated $\mathrm{Ca}_{\mathrm{v}} 1.2$, disrupted radial migration of immature cortical neurons. Removing $\mathrm{Ca}^{2+}$ permeation in the mutated $\mathrm{Ca}_{\mathrm{v}} 1.2$ abolished the migration deficit, providing the most compelling evidence obtained so far in support for a role of L-type VGCCs in radial migration. This extends previous reports in which expression of a constitutively active bacterial $\mathrm{Na}^{+}$channel, $\mathrm{NaChBac}$, caused disrupted migration that was accompanied by aberrant calcium transients (Bando et al., 2014, 2016).

L-type calcium channels are known to be involved in various fundamental neuronal processes, such as LTP, synaptic transmission, gene transcription, and cytoskeletal remodeling (Furuyashiki et al., 2002; Hofmann et al., 2014). Most research about L-type calcium channels so far has mainly focused on its physical properties or functions in adult excitable neural cells and tissues. Several GWAS and whole-exome sequencing studies suggested a significant role of $\mathrm{Ca}_{\mathrm{v}} 1.2$ as a susceptibility gene involved in several neuropsychiatric disorders (Splawski et al., 2004, 2005; Schizophrenia Psychiatric Genome-Wide Association Study (GWAS) Consortium, 2011; Cross-Disorder Group of the Psychiatric Genomics Consortium, 2013; De Rubeis et al., 2014; Purcell et al., 2014). Despite a growing interest in this issue, however, the molecular pathology that may underlie $\mathrm{Ca}_{\mathrm{v}} 1.2$ malfunction during the onset and/or progression of key neuropsychiatric diseases remains unknown. Our study provides compelling evidence that aberrant cortical wiring might be one key neurodevelopment signature of perinatal dysfunctional $\mathrm{Ca}_{\mathrm{v}} 1.2$ activity.

We specifically focused on the well established gain-of-function mutation of $\mathrm{Ca}_{\mathrm{v}} 1.2, \mathrm{G} 406 \mathrm{R}$, which was reported previously in a Timothy syndrome patient (Krey et al., 2013; Li et al., 2016). We found that this mutation provides an increase in SRCaTs and, furthermore, continuously facilitated $\mathrm{Ca}^{2+}$ entry through $\mathrm{Ca}_{\mathrm{v}} 1.2$ G406R led to significantly aberrant radial migration of cortical neurons in the layers $2 / 3$, which are known to integrate various sensory and modulatory inputs and transform this kind of information into reliable signals that are further transmitted to the layer 5 output neurons. Because radial migration is a slowly developing process in the prenatal mouse brain, we tested whether cumulative maladaptation of the migratory effects of $\mathrm{Ca}_{\mathrm{v}} 1.2$ before birth could be corrected post hoc by removing the $\mathrm{Ca}_{\mathrm{v}} 1.2-$ mediated $\mathrm{Ca}^{2+}$ overload postnatally. Our results are consistent with the idea that postnatal correction of the G406R mutation may significantly alleviate and even compensate for the prenatally acquired errors in cortical migration. Together, these results underscore hitherto unsuspected and crucial developmental impacts of perinatally expressed L-type VGCC signaling in neurodevelopment. Our data hint that adequate $\mathrm{Ca}^{2+}$ dynamics in the immature cortical circuit provide the necessary template for proper wiring across various layers of the cortex through positive and negative regulation of neurite extension and radial migration. Further experiments are now under way to test this hypothesis.

\section{References}

Ageta-Ishihara N, Takemoto-Kimura S, Nonaka M, Adachi-Morishima A, Suzuki K, Kamijo S, Fujii H, Mano T, Blaeser F, Chatila TA, Mizuno H, Hirano T, Tagawa Y, Okuno H, Bito H (2009) Control of cortical axon elongation by a GABA-driven $\mathrm{Ca} 2+$ /calmodulin-dependent protein kinase cascade. J Neurosci 29:13720-13729. CrossRef Medline

Bando Y, Irie K, Shimomura T, Umeshima H, Kushida Y, Kengaku M, Fujiyoshi Y, Hirano T, Tagawa Y (2016) Control of spontaneous Ca2 + transients is critical for neuronal maturation in the developing neocortex. Cereb Cortex 26:106-117. CrossRef Medline

Bando Y, Hirano T, Tagawa Y (2014) Dysfunction of KCNK potassium channels impairs neuronal migration in the developing mouse cerebral cortex. Cereb Cortex 24:1017-1029. CrossRef Medline

Barrett CF, Tsien RW (2008) The timothy syndrome mutation differen- 
tially affects voltage- and calcium-dependent inactivation of CaV1.2 L-type calcium channels. Proc Natl Acad Sci U S A 105:2157-2162. CrossRef Medline

Bendahhou S, Donaldson MR, Plaster NM, Tristani-Firouzi M, Fu YH, Ptácek LJ (2003) Defective potassium channel Kir2.1 trafficking underlies andersen-tawil syndrome. J Biol Chem 278:51779-51785. CrossRef Medline

Bito H, Deisseroth K, Tsien RW (1996) CREB phosphorylation and dephosphorylation: $\mathrm{a} \mathrm{Ca}(2+)$ - and stimulus duration-dependent switch for hippocampal gene expression. Cell 87:1203-1214. CrossRef Medline

Cabell L, Audesirk G (1993) Effects of selective inhibition of protein kinase C, cyclic AMP-dependent protein kinase, and $\mathrm{Ca}(2+)$-calmodulindependent protein kinase on neurite development in cultured rat hippocampal neurons. Int J Dev Neurosci 11:357-368. CrossRef Medline

Cheli VT, Santiago González DA, Namgyal Lama T, Spreuer V, Handley V, Murphy GG, Paez PM (2016) Conditional deletion of the L-type calcium channel Cav1.2 in oligodendrocyte progenitor cells affects postnatal myelination in mice. J Neurosci 36:10853-10869. CrossRef Medline

Chen Y, Stevens B, Chang J, Milbrandt J, Barres BA, Hell JW (2008) NS21: re-defined and modified supplement B27 for neuronal cultures. J Neurosci Methods 171:239-247. CrossRef Medline

Cross-Disorder Group of the Psychiatric Genomics Consortium (2013) Identification of risk loci with shared effects on five major psychiatric disorders: a genome-wide analysis. Lancet 381:1371-1379. CrossRef Medline

Deisseroth K, Bito H, Tsien RW (1996) Signaling from synapse to nucleus: postsynaptic CREB phosphorylation during multiple forms of hippocampal synaptic plasticity. Neuron 16:89-101. CrossRef Medline

De Rubeis S, De Rubeis S, He X, Goldberg AP, Poultney CS, Samocha K, Cicek AE, Kou Y, Liu L, Fromer M, Walker S, Singh T, Klei L, Kosmicki J, Shih-Chen F, Aleksic B, Biscaldi M, Bolton PF, Brownfeld JM, Cai J, Campbell NG, et al. (2014) Synaptic, transcriptional and chromatin genes disrupted in autism. Nature 515:209-215. CrossRef Medline

Dolmetsch RE, Lewis RS, Goodnow CC, Healy JI (1997) Differential activation of transcription factors induced by $\mathrm{Ca} 2+$ response amplitude and duration. Nature 386:855-858. CrossRef Medline

Fujii H, Inoue M, Okuno H, Sano Y, Takemoto-Kimura S, Kitamura K, Kano M, Bito H (2013) Nonlinear decoding and asymmetric representation of neuronal input information by CaMKIIalpha and calcineurin. Cell Rep 3:978-987. CrossRef Medline

Furuyashiki T, Arakawa Y, Takemoto-Kimura S, Bito H, Narumiya S (2002) Multiple spatiotemporal modes of actin reorganization by NMDA receptors and voltage-gated Ca2+ channels. Proc Natl Acad Sci U S A 99:1445814463. CrossRef Medline

Gomez TM, Spitzer NC (1999) In vivo regulation of axon extension and pathfinding by growth-cone calcium transients. Nature 397:350-355. CrossRef Medline

He M, Bodi I, Mikala G, Schwartz A (1997) Motif III S5 of L-type calcium channels is involved in the dihydropyridine binding site: a combined radioligand binding and electrophysiological study. J Biol Chem 272: 2629-2633. CrossRef Medline

Helton TD, Xu W, Lipscombe D (2005) Neuronal L-type calcium channels open quickly and are inhibited slowly. J Neurosci 25:10247-10251. CrossRef Medline

Henley J, Poo MM (2004) Guiding neuronal growth cones using Ca2 + signals. Trends Cell Biol 14:320-330. CrossRef Medline

Hetzenauer A, Sinnegger-Brauns MJ, Striessnig J, Singewald N (2006) Brain activation pattern induced by stimulation of L-type $\mathrm{Ca} 2+$-channels: contribution of $\mathrm{Ca}(\mathrm{V}) 1.3$ and $\mathrm{Ca}(\mathrm{V}) 1.2$ isoforms. Neuroscience 139:10051015. CrossRef Medline

Hofmann F, Flockerzi V, Kahl S, Wegener JW (2014) L-type CaV1.2 calcium channels: from in vitro findings to in vivo function. Physiol Rev 94:303-326. CrossRef Medline

Horigane S, Ageta-Ishihara N, Kamijo S, Fujii H, Okamura M, Kinoshita M, Takemoto-Kimura S, Bito H (2016) Facilitation of axon outgrowth via a Wnt5a-CaMKK-CaMKIalpha pathway during neuronal polarization. Mol Brain 9:8. CrossRef Medline

Inoue $\mathrm{M}$, Takeuchi $\mathrm{A}$, Horigane $\mathrm{S}$, Ohkura $\mathrm{M}$, Gengyo-Ando $\mathrm{K}$, Fujii $\mathrm{H}$, Kamijo S, Takemoto-Kimura S, Kano M, Nakai J, Kitamura K, Bito H (2015) Rational design of a high-affinity, fast, red calcium indicator R-CaMP2. Nat Methods 12:64-70. CrossRef Medline

Jin L, Han Z, Platisa J, Wooltorton JR, Cohen LB, Pieribone VA (2012)
Single action potentials and subthreshold electrical events imaged in neurons with a fluorescent protein voltage probe. Neuron 75:779-785. CrossRef Medline

Jing Z, Rutherford MA, Takago H, Frank T, Fejtova A, Khimich D, Moser T, Strenzke N (2013) Disruption of the presynaptic cytomatrix protein bassoon degrades ribbon anchorage, multiquantal release, and sound encoding at the hair cell afferent synapse. J Neurosci 33:4456-4467. CrossRef Medline

Kanamori T, Kanai MI, Dairyo Y, Yasunaga K, Morikawa RK, Emoto K (2013) Compartmentalized calcium transients trigger dendrite pruning in drosophila sensory neurons. Science 340:1475-1478. CrossRef Medline

Krey JF, Paşca SP, Shcheglovitov A, Yazawa M, Schwemberger R, Rasmusson $\mathrm{R}$, Dolmetsch RE (2013) Timothy syndrome is associated with activitydependent dendritic retraction in rodent and human neurons. Nat Neurosci 16:201-209. CrossRef Medline

Lautermilch NJ, Spitzer NC (2000) Regulation of calcineurin by growth cone calcium waves controls neurite extension. J Neurosci 20:315-325. CrossRef Medline

Li B, Tadross MR, Tsien RW (2016) Sequential ionic and conformational signaling by calcium channels drives neuronal gene expression. Science 351:863-867. CrossRef Medline

Lipscombe D, Pan JQ, Gray AC (2002) Functional diversity in neuronal voltage-gated calcium channels by alternative splicing of $\mathrm{Ca}(\mathrm{v})$ alphal. Mol Neurobiol 26:21-44. CrossRef Medline

Lipscombe D, Helton TD, Xu W (2004) L-type calcium channels: the low down. J Neurophysiol 92:2633-2641. CrossRef Medline

Liu Z, Ren J, Murphy TH (2003) Decoding of synaptic voltage waveforms by specific classes of recombinant high-threshold $\mathrm{Ca}(2+)$ channels. J Physiol 553:473-488. CrossRef Medline

Ma D, Tang XD, Rogers TB, Welling PA (2007) An andersen-tawil syndrome mutation in Kir2.1 (V302M) alters the G-loop cytoplasmic K+ conduction pathway. J Biol Chem 282:5781-5789. CrossRef Medline

Meijering E, Jacob M, Sarria JC, Steiner P, Hirling H, Unser M. (2004) Design and validation of a tool for neurite tracing and analysis in fluorescence microscopy images. Cytometry A 58:167-176. CrossRef Medline

Mikuni T, Nishiyama J, Sun Y, Kamasawa N, Yasuda R (2016) Highthroughput, high-resolution mapping of protein localization in mammalian brain by in vivo genome editing. Cell 165:1803-1817. CrossRef Medline

Ohkura M, Sasaki T, Sadakari J, Gengyo-Ando K, Kagawa-Nagamura Y, Kobayashi C, Ikegaya Y, Nakai J (2012) Genetically encoded green fluorescent $\mathrm{Ca} 2+$ indicators with improved detectability for neuronal $\mathrm{Ca} 2+$ signals. PLoS One 7:e51286. CrossRef Medline

Parent L, Gopalakrishnan M (1995) Glutamate substitution in repeat IV alters divalent and monovalent cation permeation in the heart $\mathrm{Ca} 2+$ channel. Biophys J 69:1801-1813. CrossRef Medline

Purcell SM, Moran JL, Fromer M, Ruderfer D, Solovieff N, Roussos P, O’Dushlaine C, Chambert K, Bergen SE, Kähler A, Duncan L, Stahl E, Genovese G, Fernández E, Collins MO, Komiyama NH, Choudhary JS, Magnusson PK, Banks E, Shakir K, et al. (2014) A polygenic burden of rare disruptive mutations in schizophrenia. Nature 506:185-190. CrossRef Medline

Seisenberger C, Specht V, Welling A, Platzer J, Pfeifer A, Kühbandner S, Striessnig J, Klugbauer N, Feil R, Hofmann F (2000) Functional embryonic cardiomyocytes after disruption of the L-type alpha1C (Cav1.2) calcium channel gene in the mouse. J Biol Chem 275:39193-39199. CrossRef Medline

Simms BA, Zamponi GW (2014) Neuronal voltage-gated calcium channels: structure, function, and dysfunction. Neuron 82:24-45. CrossRef Medline

Spitzer NC (2006) Electrical activity in early neuronal development. Nature 444:707-712. CrossRef Medline

Splawski I, Timothy KW, Sharpe LM, Decher N, Kumar P, Bloise R, Napolitano C, Schwartz PJ, Joseph RM, Condouris K, Tager-Flusberg H, Priori SG, Sanguinetti MC, Keating MT (2004) Ca(V)1.2 calcium channel dysfunction causes a multisystem disorder including arrhythmia and autism. Cell 119:19-31. CrossRef Medline

Splawski I, Timothy KW, Decher N, Kumar P, Sachse FB, Beggs AH, Sanguinetti MC, Keating MT (2005) Severe arrhythmia disorder caused by cardiac L-type calcium channel mutations. Proc Natl Acad Sci U S A 102: 8089-8096; discussion 8086-8088. CrossRef Medline

Striessnig J, Koschak A, Sinnegger-Brauns MJ, Hetzenauer A, Nguyen NK, Busquet P, Pelster G, Singewald N (2006) Role of voltage-gated L-type 
$\mathrm{Ca} 2+$ channel isoforms for brain function. Biochem Soc Trans 34:903909. CrossRef Medline

Tabata H, Nakajima K (2001) Efficient in utero gene transfer system to the developing mouse brain using electroporation: visualization of neuronal migration in the developing cortex. Neuroscience 103:865-872. CrossRef Medline

Takemoto-Kimura S, Ageta-Ishihara N, Nonaka M, Adachi-Morishima A, Mano T, Okamura M, Fujii H, Fuse T, Hoshino M, Suzuki S, Kojima M, Mishina M, Okuno H, Bito H (2007) Regulation of dendritogenesis via a lipid-raft-associated Ca2+/calmodulin-dependent protein kinase CLICKIII/CaMKIgamma. Neuron 54:755-770. CrossRef Medline

Takemoto-Kimura S, Suzuki K, Kamijo S, Ageta-Ishihara N, Fujii H, Okuno $\mathrm{H}$, Bito H (2010) Differential roles for CaM kinases in mediating excitation-morphogenesis coupling during formation and maturation of neuronal circuits. Eur J Neurosci 32:224-230. CrossRef Medline

Tang F, Dent EW, Kalil K (2003) Spontaneous calcium transients in developing cortical neurons regulate axon outgrowth. J Neurosci 23:927-936. CrossRef Medline

Schizophrenia Psychiatric Genome-Wide Association Study (GWAS) Consortium (2011) Genome-wide association study identifies five new schizophrenia loci. Nat Genet 43:969-976. CrossRef Medline

Vacher H, Mohapatra DP, Trimmer JS (2008) Localization and targeting of voltage-dependent ion channels in mammalian central neurons. Physiol Rev 88:1407-1447. CrossRef Medline

Wang X, Schwarz TL (2009) The mechanism of Ca2+-dependent regulation of kinesin-mediated mitochondrial motility. Cell 136:163-174. CrossRef Medline

Wayman GA, Lee YS, Tokumitsu H, Silva A, Soderling TR (2008) Calmodulin-kinases: modulators of neuronal development and plasticity. Neuron 59:914-931. CrossRef Medline

Wheeler DG, Groth RD, Ma H, Barrett CF, Owen SF, Safa P, Tsien RW (2012) $\mathrm{Ca}(\mathrm{V}) 1$ and $\mathrm{Ca}(\mathrm{V}) 2$ channels engage distinct modes of $\mathrm{Ca}(2+)$ signaling to control CREB-dependent gene expression. Cell 149:11121124. CrossRef Medline

Xu W, Lipscombe D (2001) Neuronal Ca(V)1.3alpha(1) L-type channels activate at relatively hyperpolarized membrane potentials and are incompletely inhibited by dihydropyridines. J Neurosci 21:5944-5951. CrossRef Medline

Zhang H, Fu Y, Altier C, Platzer J, Surmeier DJ, Bezprozvanny I (2006) Ca1.2 and CaV1.3 neuronal L-type calcium channels: differential targeting and signaling to pCREB. Eur J Neurosci 23:2297-2310. CrossRef Medline

Zheng JQ (2000) Turning of nerve growth cones induced by localized increases in intracellular calcium ions. Nature 403:89-93. CrossRef Medline 